\title{
Local BDNF Delivery to the Injured Cervical Spinal Cord using an Engineered Hydrogel Enhances Diaphragmatic Respiratory Function
}

\author{
[Diswarup Ghosh, ${ }^{1 *}$ Zhicheng Wang, ${ }^{2 *}$ Jia Nong, ${ }^{2}$ Mark W. Urban, ${ }^{1}$ Zhiling Zhang, ${ }^{2}$ Victoria A. Trovillion, ${ }^{1}$ \\ Megan C. Wright, ${ }^{3}{ }^{\circ}$ Yinghui Zhong, ${ }^{2}$ and Angelo C. Lepore ${ }^{1}$ \\ ${ }^{1}$ Department of Neuroscience, Vickie and Jack Farber Institute for Neuroscience, Sidney Kimmel Medical College at Thomas Jefferson University, \\ Philadelphia, Pennsylvania 19107, 2School of Biomedical Engineering, Science and Health Systems, Drexel University, Philadelphia, Pennsylvania 19104, \\ and ${ }^{3}$ Department of Biology, Arcadia University, Glenside, Pennsylvania 19038
}

We developed an innovative biomaterial-based approach to repair the critical neural circuitry that controls diaphragm activation by locally delivering brain-derived neurotrophic factor (BDNF) to injured cervical spinal cord. BDNF can be used to restore respiratory function via a number of potential repair mechanisms; however, widespread BDNF biodistribution resulting from delivery methods such as systemic injection or lumbar puncture can lead to inefficient drug delivery and adverse side effects. As a viable alternative, we developed a novel hydrogel-based system loaded with polysaccharide-BDNF particles self-assembled by electrostatic interactions that can be safely implanted in the intrathecal space for achieving local BDNF delivery with controlled dosing and duration. Implantation of BDNF hydrogel after $\mathrm{C} 4 / \mathrm{C} 5$ contusion-type spinal cord injury (SCI) in female rats robustly preserved diaphragm function, as assessed by in vivo recordings of compound muscle action potential and electromyography amplitudes. However, BDNF hydrogel did not decrease lesion size or degeneration of cervical motor neuron soma, suggesting that its therapeutic mechanism of action was not neuroprotection within spinal cord. Interestingly, BDNF hydrogel significantly preserved diaphragm innervation by phrenic motor neurons (PhMNs), as assessed by detailed neuromuscular junction morphological analysis and retrograde PhMN labeling from diaphragm using cholera toxin B. Furthermore, BDNF hydrogel enhanced the serotonergic axon innervation of PhMNs that plays an important role in modulating PhMN excitability. Our findings demonstrate that local BDNF hydrogel delivery is a robustly effective and safe strategy to restore diaphragm function after SCI. In addition, we demonstrate novel therapeutic mechanisms by which BDNF can repair respiratory neural circuitry.

Key words: biomaterial; contusion; diaphragm; phrenic motor neuron; respiratory; spinal cord injury

\section{Significance Statement}

Respiratory compromise is a leading cause of morbidity and mortality following traumatic spinal cord injury (SCI). We used an innovative biomaterial-based drug delivery system in the form of a hydrogel that can be safely injected into the intrathecal space for achieving local delivery of brain-derived neurotrophic factor (BDNF) with controlled dosing and duration, while avoiding side effects associated with other delivery methods. In a clinically relevant rat model of cervical contusion-type SCI, BDNF hydrogel robustly and persistently improved diaphragmatic respiratory function by enhancing phrenic motor neuron (PhMN) innervation of the diaphragm neuromuscular junction and by increasing serotonergic innervation of PhMNs in ventral horn of the cervical spinal cord. These exciting findings demonstrate that local BDNF hydrogel delivery is a safe and robustly effective strategy to maintain respiratory function after cervical SCI.

\section{Introduction}

Respiratory dysfunction is a major problem following cervical spinal cord injury

Pulmonary compromise is a leading cause of mortality and morbidity after SCI, both acutely and at chronic stages (Shanmuga- nathan et al., 2008). There is decreased life expectancy for ventilator-dependent patients, often due to respiratory infections. Limited spontaneous respiratory recovery can occur after spinal cord injury (SCI); however, this response is often insufficient to allow patients to regain significant breathing function or 
to perform other important pulmonary tasks such as coughing (which can result in respiratory infection; Warren and Alilain, 2014). Importantly, cervical SCI represents greater than half of all human cases, in addition to often resulting in the most severe physical and psychological debilitation (Lane et al., 2008). Cervical trauma disrupts the critical neural circuitry that controls a number of respiratory muscles, in particular the diaphragm (Warren et al., 2014).

\section{Diaphragm dysfunction plays a central role in respiratory compromise after cervical SCI}

Compromised inspiratory breathing occurs in a significant percentage of SCI patients due to paralysis of the major inspiratory muscle, the diaphragm (but likely also via effects on other muscles; Strakowski et al., 2007). The diaphragm is innervated by phrenic motor neurons (PhMNs) located at cervical levels 3-5 (Lane et al., 2009). PhMN output is driven by descending premotor bulbospinal neurons in a brainstem nucleus called the rostral ventral respiratory group (rVRG; Zimmer et al., 2007). Cervical SCI results in diaphragmatic respiratory compromise due to (1) $\mathrm{PhMN}$ loss and consequent denervation at the diaphragm neuromuscular junction (NMJ), and (2) injury to descending bulbospinal respiratory axons and consequent silencing of spared PhMNs.

\section{Brain-derived neurotrophic factor hydrogel delivered locally to cervical SCI is a clinically applicable approach to repair respiratory neural circuitry}

We chose to deliver brain-derived neurotrophic factor (BDNF)

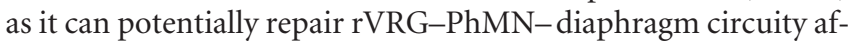
ter cervical contusion via a number of therapeutic mechanisms, including the following: (1) reducing secondary degeneration of this neural circuit that occurs following initial trauma (Novikova et al., 2000); (2) promoting regrowth of injured rVRG axons and/or sprouting of spared rVRG fibers (Weishaupt et al., 2012); (3) acting as a guidance molecule to direct rVRG axon reinnervation of their appropriate PhMN targets (Weishaupt et al., 2012); (4) modulating PhMN properties such as dendritic morphology, intrinsic excitability, glutamate receptor expression profile (Gill et al., 2016), and activation in response to excitatory synaptic input (Dale-Nagle et al., 2010); (5) enhancing synaptic transmission at the diaphragm NMJ (Mantilla et al., 2004; Gransee et al., 2013); and (6) maintaining diaphragm NMJ innervation and/or stimulating morphological changes at the NMJ such as terminal sprouting of phrenic motor axons and reinnervation of denervated endplates (Pitts et al., 2006). We have attempted to harness the multifaceted therapeutic potential of BDNF to promote repair of the critical neural circuitry controlling diaphragm function. In this study, we have specifically assessed the ability of BDNF to modulate (1) diaphragm innervation by phrenic motor axons peripherally at the NMJ and (2) PhMN innervation by descending serotonergic input centrally within the ventral horn, given that they are both critical to diaphragm activation and that the effects of BDNF on these mechanisms have not been assessed

\section{*B.G. and Z.W. contributed equally to this work.}

The authors declare no competing financial interests.

Correspondence should be addressed to either of the following: Dr. Angelo C. Lepore, Department of Neuroscience, Vickie and Jack Farber Institute for Neuroscience, Sidney Kimmel Medical College at Thomas Jefferson University, Philadelphia, PA 19107, E-mail: angelo.lepore@jefferson.edu; or Dr. Yinghui Zhong, School of Biomedical Engineering, Science and Health Systems, Drexel University, Bossone 7-716, 3141 Chestnut Street, Philadelphia, PA 19104, E-mail: yz348@drexel.edu.

DOI:10.1523/JNEUROSCI.3084-17.2018

Copyright $\odot 2018$ the authors $\quad 0270-6474 / 18 / 385983-14 \$ 15.00 / 0$ in the context of respiratory circuitry after SCI. Importantly, a number of neuronal components of the rVRG-PhMN-diaphragm circuit express the tropomyosin-related kinase receptor subtype B (TrkB) receptor, including PhMNs (Mantilla et al., 2013, 2014a) and serotonergic raphe neurons (Goswami et al., 2010). We have also found that rVRG neurons express TrkB (B. Charsar and A. Lepore unpublished observations), suggesting that BDNF hydrogel could also act upon these neurons to induce, for example, plasticity within the cervical spinal cord such as the regrowth of injured rVRG axons and/or sprouting of spared rVRG fibers to increase descending bulbospinal input to the PhMN pool.

\section{Hydrogel-based system allows for safe delivery of BDNF to cervical SCI}

Both intrathecal injection of BDNF solution (Mantilla et al., 2013; Hernandez-Torres et al., 2017) and intraspinal transplantation of BDNF-expressing mesenchymal stem cells (Gransee et al., 2015) partially improve diaphragm muscle activity in rodent models of cervical SCI. Unlike the targeted local delivery achieved with our system, clinically used lumbar intrathecal injection can result in more widespread distribution, and strategies such as viral delivery and cell transplantation may result in persistent expression, which potentially reduces its efficacy. Previous studies have also reported that persistent, anatomically widespread distribution of high levels of BDNF in the spinal cord can induce unwanted side effects such as neuropathic pain, hyperreflexia, and abnormal limb motor function that prevent its therapeutic use (Boyce and Mendell, 2014). Furthermore, clinical trials for nervous system diseases using systemic delivery of various neurotrophic factors have been associated with both side effects and lack of therapeutic efficacy (Miller et al., 1996). To address these important issues, we have developed a biocompatible drug delivery system in the form of a hydrogel loaded with polysaccharideBDNF particles self-assembled by electrostatic interactions. We have used this biomaterial-based platform to locally deliver BDNF to the injured cervical spinal cord with controlled duration to effectively repair $\mathrm{rVRG}-\mathrm{PhMN}$-diaphragm circuitry and consequently rescue diaphragmatic respiratory function with minimized side effects.

\section{Materials and Methods}

Animals. Female Sprague Dawley rats weighing 250-300 g were purchased from Taconic Farms. All animals were housed in a humidity-, temperature-, and light-controlled animal facility with ad libitum access to water and food. All experimental procedures were performed in compliance with protocols approved by the Thomas Jefferson University Institutional Animal Care and Use Committee (IACUC) and the Drexel University IACUC, as well as in accordance with the Society for Neuroscience "Policies on the Use of Animals and Humans in Neuroscience Research."

Unilateral cervical contusion model. We used only female rats as we extensively optimized and comprehensively characterized the unilateral cervical contusion SCI model both functionally and histologically in female rats in our previous work (Nicaise et al., 2012, 2013). This contusion paradigm results in robust secondary $\mathrm{PhMN}$ degeneration, interruption of descending axonal populations that innervate the PhMN pool, and persistent diaphragm dysfunction. Briefly, cervical contusion was performed on the right side of spinal cord. Rats were deeply anesthetized with an intraperitoneal injection of a cocktail of ketamine $(100 \mathrm{mg} / \mathrm{kg})$, xylazine ( $5 \mathrm{mg} / \mathrm{kg}$ ), and acepromazine ( $2 \mathrm{mg} / \mathrm{kg}$ ). The cervical dorsal skin and underlying muscles were incised. The paravertebral muscles overlying $\mathrm{C} 4-\mathrm{C} 5$ were removed. At level C4/C5, rats were then subjected to a contusion using the Infinite Horizon Spinal Impactor (Precision Systems and Instrumentation) using a $1.5 \mathrm{~mm}$ tip at a force of $395 \mathrm{kdyn}$. 
Formulation and administration of hydrogels. Dextran sulfate (DS; Sigma-Aldrich) and BDNF (PeproTech) were dissolved in deionized water at final concentrations of 4 and $0.8 \mathrm{mg} / \mathrm{ml}$, respectively. The $4 \mathrm{mg} / \mathrm{ml}$ chitosan $(\mathrm{CH})$ solution was prepared in $0.6 \%(\mathrm{v} / \mathrm{v})$ acetic acid. Ten microliters of DS and BDNF solution was then mixed with $4 \mathrm{mg} / \mathrm{ml} \mathrm{CH}$ in equal volume to form self-assembled DS-CH-BDNF complexes (particles). After centrifugation at $10,000 \mathrm{rpm}$ for $10 \mathrm{~min}$ to remove supernatant, the complexes were loaded in $20 \mu \mathrm{l}$ of $1.5 \%$ (w/v) agarose hydrogel. Immediately after the contusion SCI, $20 \mu \mathrm{l}$ of agarose hydrogel loaded with DS-CH-BDNF complexes was administered into the intrathecal space at the injury site. Another $100 \mu \mathrm{l}$ of blank agarose hydrogel was applied epidurally to protect the injury site.

Characterization of BDNF release. To measure BDNF release, rhodamine was conjugated to BDNF following a protocol provided by the Pierce Rhodamine Protein Labeling Kit (Thermo Fisher Scientific). A mixture of equal amounts of rhodamine-BDNF and BDNF was used to fabricate the drug delivery system following the procedures described above. Agarose hydrogel loaded with DS-CH-BDNF particles was incubated in HBSS, $\mathrm{pH} 7.4$, at $37^{\circ} \mathrm{C}$ for quantification of BDNF release. The release media were changed every $24 \mathrm{~h}$. The amount of BDNF released every $24 \mathrm{~h}$ was quantified by measuring at an excitation wavelength of $552 \mathrm{~nm}$ and an emission wavelength of $575 \mathrm{~nm}$.

Bioactivity of released BDNF. Rat cortical neurons were used to assess the bioactivity of released BDNF. The cortices were isolated from embryonic day 17 rat embryos in accordance with protocols approved by the Drexel University IACUC. Dissociated cortical cells were seeded at a density of 120,000 cells $/ \mathrm{cm}^{2}$ and maintained in Neurobasal medium supplemented with 2\% B27 supplement and 2 mm L-glutamine. After $7 \mathrm{~d}$, B27 supplement in the culture medium was replaced by B27 minus antioxidants (AOs) to remove the neuroprotective AOs from the culture medium. The neurons were then treated with $100 \mu \mathrm{M}$ hydrogen peroxide $\left(\mathrm{H}_{2} \mathrm{O}_{2}\right) ; 100 \mu \mathrm{M} \mathrm{H} \mathrm{O}_{2}$ supplemented with equal amounts of fresh BDNF and rhodamine-BDNF at a total concentration of $30 \mathrm{ng} / \mathrm{ml}$, with or without $120 \mathrm{ng} / \mathrm{ml}$ anti-BDNF blocking antibody (PeproTech); $100 \mu \mathrm{M}$ $\mathrm{H}_{2} \mathrm{O}_{2}$ supplemented with BDNF released during a $24 \mathrm{~h}$ period on day 17 (containing a mixture of BDNF and rhodamine-BDNF, which was diluted to $30 \mathrm{ng} / \mathrm{ml}$ ), with or without $120 \mathrm{ng} / \mathrm{ml}$ anti-BDNF blocking antibody; or $100 \mu \mathrm{M} \mathrm{H} \mathrm{H}_{2} \mathrm{O}_{2}$ supplemented with release medium from hydrogel loaded with DS-CH particles without BDNF. Untreated neurons were used as a negative control. After $6 \mathrm{~h}$, cell viability was quantified using the alamarBlue cell viability assay. The cells were then stained with $2 \mu \mathrm{M}$ Calcein AM (live staining) and imaged with an inverted fluorescence microscope (Leica).

Diaphragm compound muscle action potential measurements. Rats were anesthetized with isoflurane at a concentration of $2.0-2.5 \%$. Phrenic nerve conduction studies were performed with single stimulation $(0.5 \mathrm{~ms}$ duration; $6 \mathrm{mV}$ amplitude) at the neck (either ipsilateral or contralateral to the contusion site) close to the passage of the phrenic nerve (Lepore et al., 2010; Li et al., 2014), and a ground needle electrode was inserted into the tail. A reference electrode was placed in the right abdominal region subcutaneously. Reproducibility was ensured by recording 10-20 times with a $5 \mathrm{~s}$ interval between stimulations. Recording was obtained via a surface strip along the costal margin of the diaphragm, and compound muscle action potential (CMAP) amplitude was measured from baseline to peak. Recordings were made using an PowerLab 8/30 Stimulator and a BioAmp Amplifier (ADInstruments), followed by computer-assisted data analysis (Scope 3.5.6, ADInstruments; RRID:SCR_001620). CMAPs were measured three times for each animal at 1 week intervals following SCI/treatment.

EMG recordings. Animals were anesthetized with isoflurane at a concentration of $3.0 \%$. Once deeply anesthetized, a laparotomy was performed to expose the hemidiaphragm ipsilateral or contralateral to the contusion. Bipolar electrodes spaced by $3 \mathrm{~mm}$ were inserted into specific subregions of the hemidiaphragm (i.e., dorsal, medial, or ventral subregions; Li et al., 2015). Recordings were averaged over a 2 min time frame for each animal, and peak amplitude, burst duration, and frequency were measured. Using LabChart 7 software (ADInstruments; RRID:SCR_001620), the EMG signal was amplified and filtered through a bandpass filter $(50-3000 \mathrm{~Hz})$.
EMG recordings were conducted once per animal immediately before the animal was killed.

Retrograde labeling of PhMNs in cervical spinal cord. Two weeks before the animal was killed, cholera toxin B subunit (CTB) conjugated to Alexa Fluor 647 (Life Technologies) was injected unilaterally into the intrapleural space of the ipsilateral hemidiaphragm under anesthesia (Nicaise et al., 2012; Li et al., 2014). This procedure was conducted to specifically label PhMN soma/dendrites in the ventral horn of the cervical spinal cord. In brief, following laparotomy, $15 \mu \mathrm{l}$ of CTB $(0.2 \%$ solution in distilled water) was intrapleurally delivered transdiaphragmatically (3 injections of $5 \mu \mathrm{l}$ each) using a $20 \mu \mathrm{l}$ Hamilton syringe. Abdominal muscles and skin were then sutured separately.

Perfusion and spinal cord dissection. Animals were killed with a mixture of ketamine $(300 \mathrm{mg} / \mathrm{kg})$, xylazine $(15 \mathrm{mg} / \mathrm{kg})$, and acepromazine $(6$ $\mathrm{mg} / \mathrm{kg}$ ). After the hemidiaphragm was dissected, the animals were perfused using $0.9 \%$ saline solution, followed by $4 \%$ paraformaldehyde solution. Spinal cord and brain were then removed and postfixed in a $4 \%$ paraformaldehyde solution overnight at $4^{\circ} \mathrm{C}$. After cryoprotection in $30 \%$ sucrose for $\sim 3 \mathrm{~d}$, cervical spinal cords were embedded in tissuefreezing medium and then frozen in dry ice. Spinal cord sections were cut (in both transverse and sagittal orientations) at a thickness of $20 \mu \mathrm{m}$.

Assessment of lesion volume and ventral horn motor neurons. Transverse spinal cord sections $160 \mu \mathrm{m}$ apart were stained with cresyl violet for Nissl bodies and Eriochrome cyanine for myelin. The lesion area on each section was quantified using ImageJ software (RRID:SCR_003070) to calculate the total lesion volume. Motor neurons were outlined in Nissl myelin-stained sections in the cervical spinal cord ventral horn. Motor neuron cell bodies with a clearly identifiable nucleolus and a soma of $>200 \mu \mathrm{m}^{2}$ were counted in a blinded manner (Lepore et al., 2011). PhMNs in the C3-C5 ventral horn specifically labeled with CTB were also counted in transverse sections spaced $160 \mu \mathrm{m}$ apart in a blinded manner.

Neuromuscular junction staining. Fresh hemidiaphragm was dissected before perfusion for whole-mount immunohistochemistry (Wright et al., 2007; Li et al., 2014). Briefly, muscle was stretched, pinned down to Sylgard medium (Thermo Fisher Scientific), and extensively cleaned to remove any connective tissue to allow for antibody penetration. Motor axons and their terminals were labeled with primary antibodies SMI312R (Covance; RRID:AB_2314906) and SV2-s (Developmental Studies Hybridoma Bank at the University of Iowa, Iowa City, IA; RRID: AB_2315387), respectively, and both antibodies were detected with FITC anti-mouse IgG secondary (Jackson ImmunoResearch). Postsynaptic acetylcholine receptors were labeled with rhodamine-conjugated $\alpha$-bungarotoxin (Life Technologies). Diaphragms were then mounted with VECTASHIELD mounting medium (Vector Laboratories), coverslipped, and stored at $-20^{\circ} \mathrm{C}$. Labeled muscles were then analyzed for total numbers of intact, completely denervated and partially denervated NMJs. Muscle was imaged on a FluoView FV1000 Confocal Microscope (Olympus; RRID:SCR_014215). We conducted NMJ staining only in ipsilateral hemidiaphragm because in our previous study we did not observe significant denervation or sprouting in contralateral hemidiaphragm after unilateral cervical contusion SCI (Nicaise et al., 2012).

Immunohistochemistry. Frozen transverse spinal cord sections were air dried, washed, and finally permeabilized with $0.4 \%$ Triton X-100 in PBS for $5 \mathrm{~min}$ at room temperature. For immunohistochemistry of 5-HT and the presynaptic marker Synaptophysin, samples were incubated in blocking solution (PBS containing 5\% normal goat serum, $1 \%$ Triton X-100, and $0.05 \%$ Tween 20 ) for $2 \mathrm{~h}$ at room temperature. Sections were labeled overnight at $4^{\circ} \mathrm{C}$ with mouse synaptophysin antibody (1:250; Abcam; RRID:AB_2198854), and rabbit 5-HT antibody (1:15,000; Immunostar; RRID:AB_572263) in blocking solution. Sections were then washed three times with PBS ( $5 \mathrm{~min} /$ wash) and incubated with Alexa Fluor 405 goatanti-mouse (1:200; Abcam) and Alexa Fluor 488 goat-anti-rabbit (1:200; Jackson ImmunoResearch) in blocking solution for $2 \mathrm{~h}$ at room temperature. After washing three times with PBS ( $5 \mathrm{~min} /$ wash), sections were coverslipped. Images were acquired with a Zeiss Imager M2 Upright Microscope or on a FluoView FV1000 Confocal Microscope (Olympus), and MetaMorph software (Molecular Devices; RRID:SCR_002368) was used for quantification. 


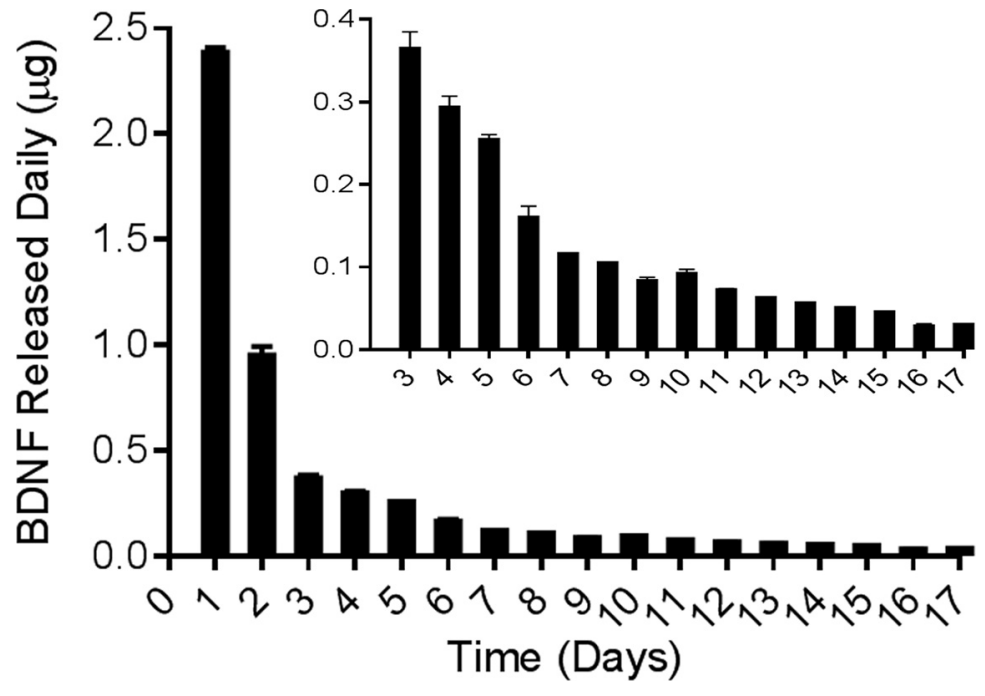

Figure 1. BDNF was released from hydrogel loaded with DS-CH-BDNF complexes for $>17 \mathrm{~d}$. Inset depicts release profile from 3 to 17 d. $n=3 /$ group.

Quantification of 5-HT immunohistochemistry. For quantitative analysis of 5-HT immunostaining (see Fig. 9), we used the same image acquisition parameters for all samples, including excitation light intensity, camera settings, and exposure time. In transverse sections, we created concentric circles of increasing radii of $50 \mu \mathrm{m}$ measured from the $\mathrm{CTB}^{+}$ $\mathrm{PhMN}$ location. We quantified the number of 5-HT axon profiles, total 5-HT axonal length, and integrated intensity of 5-HT immunolabeling at each distance $(0-50,50-100$, and $100-150 \mu \mathrm{m})$. For quantification of putative synaptic contacts between $5-\mathrm{HT}^{+} /$synaptophysin ${ }^{+}$terminals and CTB-labeled PhMNs, we considered those contacts within $2.5 \mu \mathrm{m}$ of the surface of the $\mathrm{CTB}^{+} \mathrm{PhMN}$ in single- $z$ confocal images, based on previous work (Kinkead et al., 1998; Issa et al., 2010).

Experimental design and statistical analysis. In the Results section, we provide details of group means, SEM, statistical tests used, and results of statistical analyses (including the exact $p$ value) for each experiment.

We gave careful thought to minimizing animal numbers. To conduct an a priori power analysis (with SigmaPlot 12) for studies involving in vivo diaphragm functional analysis, spinal cord histology, spinal cord immunohistochemistry, and whole-mount muscle labeling, we used previously obtained values (i.e., EMG and CMAP recordings, lesion size analysis, motor neuron counts, CTB labeling, NMJ morphology assessment, and 5-HT axon quantification) from our laboratories for rats subjected to this same cervical contusion model to generate an estimate of SD.

We conducted all surgical procedures, as well as all quantitative histological and functional analyses, in a blinded fashion. The surgeon conducting the contusion and hydrogel injection were blinded to the experimental group identity of a given animal throughout the study. In addition, the experimenters conducting EMG and CMAP recordings did not know the group identity of the animals being assessed. Furthermore, experimenters were blinded to the sample identity during subsequent quantitative analysis of these functional assays, as well as while conducting all histological analyses following the killing of an animal. Before starting a study, rats were randomly assigned to experimental groups, and the different hydrogels used within a given experiment were randomly distributed across these rats. In order for an animal to be included in functional and histological quantification, we verified using lesion analysis that the contusion injury was properly located at the appropriate spinal cord level and that the injury was targeted to just the right side of the spinal cord.

Results were expressed as the mean \pm SEM. An unpaired $t$ test was used to assess statistical significance between two groups. With respect to multiple comparisons involving three or more groups, statistical significance was assessed by ANOVA. Statistics were computed with GraphPad Prism 5 (GraphPad Software). A $p$ value $<0.05$ was considered as statistically significant.

\section{Results}

Bioactive BDNF was released from agarose hydrogel loaded with DS-CHBDNF complexes for $>17 \mathrm{~d}$

In this study, we found that negatively charged DS could form self-assembled complexes (particles) with positively charged $\mathrm{CH}$ and BDNF possibly through electrostatic interaction. We embedded these particles in agarose hydrogel so that they can remain localized at the injury site in vivo. Relatively high amounts of BDNF were released from BDNF-loaded hydrogel in vitro in the first $2 \mathrm{~d}$, followed by low-dose release $(364.2-29.5 \mathrm{ng} / \mathrm{d})$ for $>15$ d (Fig. 1 ). We further investigated whether this novel drug delivery system is capable of maintaining the bioactivity of encapsulated BDNF. $\mathrm{H}_{2} \mathrm{O}_{2}$-induced massive loss of cortical neurons in vitro $(18.29 \pm 0.80 \%$ viability vs $100 \pm 2.23 \%$ for untreated control; Fig. $2 A, B$ ), whereas fresh BDNF (38.94 $\pm 1.38 \%$; Fig. $2 D$ ) or BDNF released from the hydrogel on day 17 (38.36 $\pm 1.13 \%$; Fig. $2 E$; diluted to the same concentration as fresh BDNF) improved neuron survival $\left(F_{(6,114)}\right.$ $=427.1$, Tukey's post-test; $\mathrm{H}_{2} \mathrm{O}_{2}$ vs fresh BDNF, $p<0.0001$; $\mathrm{H}_{2} \mathrm{O}_{2}$ vs released BDNF, $p<0.0001$; released without BDNF vs fresh BDNF, $p<0.0001$; released without BDNF vs released BDNF, $p<0.0001$; ANOVA; $n=3$ /group). Anti-BDNF antibody completely blocked the neuroprotective effect of both fresh (18.76 \pm 1.13\%; Fig. $2 F)$ and hydrogel-released (17.53 $\pm 0.47 \%$; Fig. $2 G) \operatorname{BDNF}\left(F_{(6,114)}=427.1\right.$, Tukey's post-test; fresh BDNF vs fresh $\mathrm{BDNF}+\mathrm{Ab}, p<0.0001$; released $\mathrm{BDNF}$ vs released $\mathrm{BDNF}$ $+\mathrm{Ab}, p<0.0001$; ANOVA; $n=3$ /group), suggesting both that the neuroprotective effect was from BDNF and that this drug delivery system itself was not neuroprotective. Quantitative cell viability assessment confirmed that both fresh and hydrogelreleased BDNF significantly inhibited $\mathrm{H}_{2} \mathrm{O}_{2}$-induced neurotoxicity (Fig. $2 \mathrm{H}$ ). Moreover, there was no significant difference in cell viability between neuronal cultures treated with fresh and hydrogel-released BDNF, suggesting that the polysaccharidebased BDNF delivery system maintained the activity of BDNF for at least $17 \mathrm{~d}$. In addition, release medium from agarose hydrogel loaded with DS-CH particles without BDNF showed no neuroprotective or neurotoxic effect (17.53 $\pm 0.47 \%$; Fig. $2 C)$; this result is consistent with that of the anti-BDNF antibody blocking test and confirms the safety of this drug delivery system.

\section{BDNF hydrogel implantation significantly improved diaphragm function following cervical contusion SCI} In a rat model of unilateral C4/C5 contusion SCI, we subdurally implanted BDNF hydrogel or blank hydrogel control at the level of the contusion immediately postinjury. We tested the in vivo effects of hydrogel implantation on diaphragmatic respiratory function by quantifiably measuring both inspiratory EMGs and evoked CMAPs in anesthetized rats.

We assessed EMG burst amplitude as a measure of rhythmic diaphragm activation under normal eupnic breathing conditions. We conducted intradiaphragm EMG recordings separately from ventral, medial, and dorsal subregions of the hemidiaphragm ipsilateral to the contusion. We and others have previously shown that each diaphragm subregion is innervated by $\mathrm{PhMNs}$ whose cell bodies are located at different rostral-caudal 

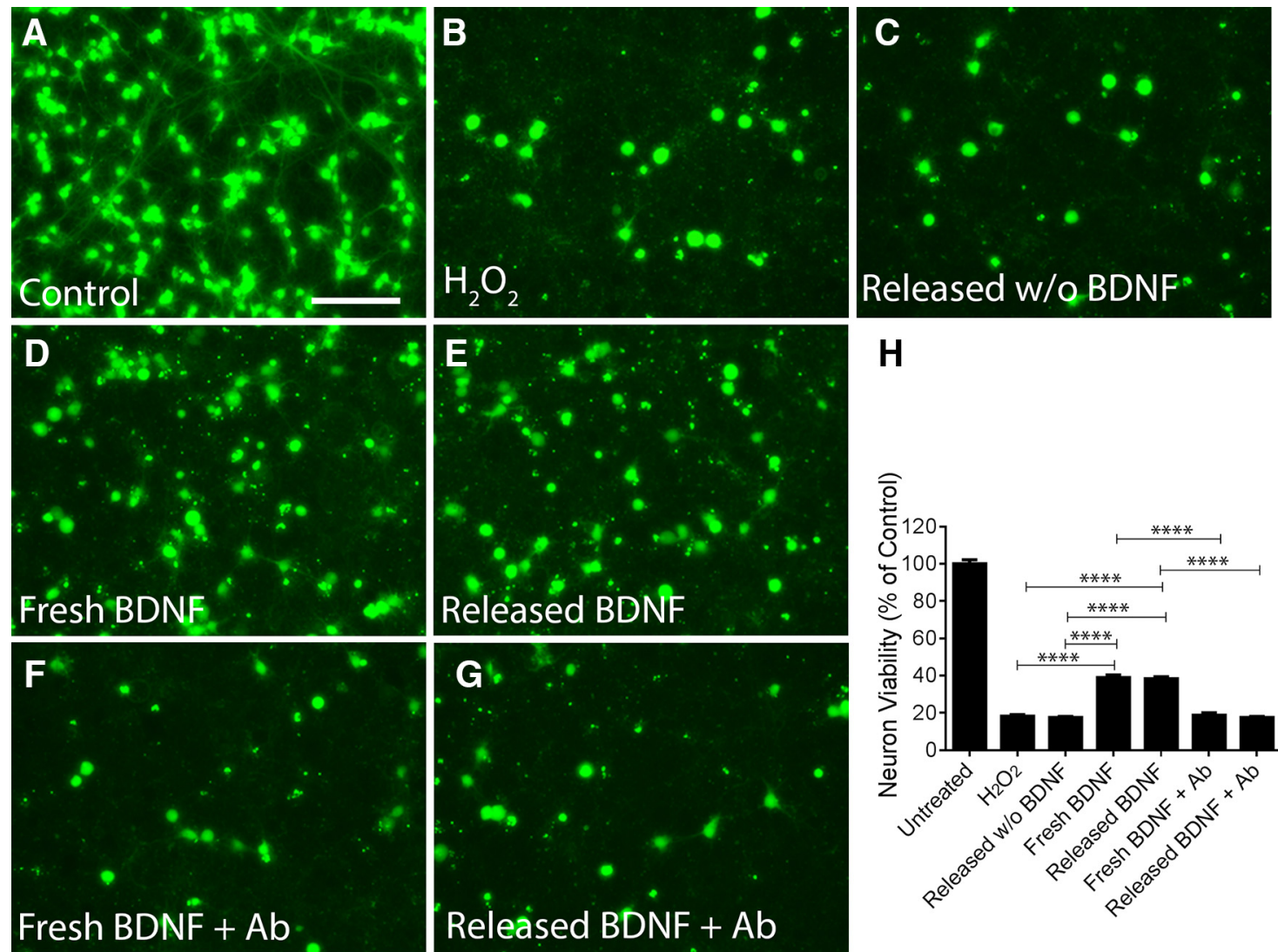

H

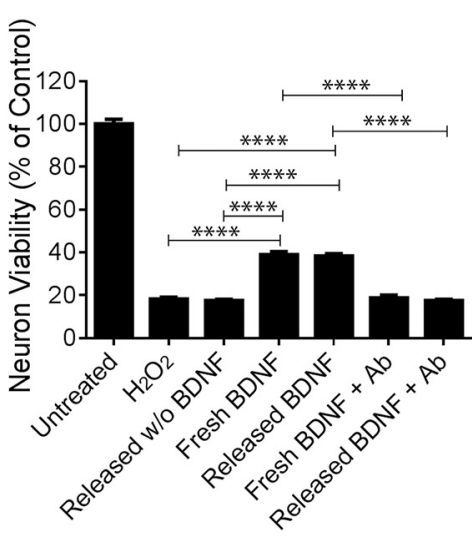

Figure 2. Released BDNF maintained bioactivity throughout the duration of release. $A-G$, Representative images showing the morphology of cultured cortical neurons stained with calcein AM. Scale bar, $100 \mu \mathrm{m}$. $\boldsymbol{H}$, Viability under different treatments. We expressed results as the mean \pm SEM. ${ }^{* * *} p<0.0001$.

portions of the C3-C5 spinal cord ( $\mathrm{Li}$ et al., 2015). In contusion animals implanted with blank hydrogel, we observed the largest loss of EMG amplitude in the dorsal subregion of the muscle $(1.7 \pm 0.34 \mathrm{vs} 8.16 \pm 0.83 \mathrm{mV} / \mathrm{s}$ for the intact contralateral hemidiaphragm; Fig. $3 A, C$ ), which is primarily innervated by the most caudal portion of the PhMN pool. Importantly, we performed the contusion at $\mathrm{C} 4 / \mathrm{C} 5$, demonstrating that the portion of the PhMN pool most severely affected by the injury matched the subregion of the hemidiaphragm with the lowest EMG amplitudes. EMG amplitudes in the ventral and medial subregions of the muscle were not as severely reduced compared with EMG amplitudes obtained from these same subregions in the control uninjured condition (Fig. $3 C$ ), particularly in the ventral subregion, which is primarily innervated by the most rostrally located PhMNs (i.e., on the opposite end from the contusion). In addition, BDNF showed no effect on EMG amplitudes at these ventral (blank gel, $4.89 \pm 0.70 \mathrm{mV} / \mathrm{s}$; BDNF, $4.59 \pm 0.34 \mathrm{mV} / \mathrm{s}$ ) and medial (blank gel, $2.96 \pm 1.10 \mathrm{mV} / \mathrm{s}$; BDNF, $3.04 \pm 0.13 \mathrm{mV} / \mathrm{s}$ ) hemidiaphragm locations compared with blank hydrogel control $\left(F_{(6,21)}=10.47\right.$; Tukey's post-test; ventral: blank vs BDNF, $p=$ 0.9 ; medial: blank vs BDNF, $p=0.9$; contralateral vs ventral/ blank, $p=0.06$; contralateral vs ventral/BDNF, $p=0.06$; contralateral vs medial/blank, $p=0.0004$; contralateral vs medial/ BDNF, $p=0.0015$; ANOVA; $n=4$ rats/group; Fig. $3 C$ ). Compared with blank hydrogel-implanted animals (Fig. 3A), BDNF hydrogel resulted in significantly greater diaphragm EMG amplitudes in the dorsal subregion (blank gel, $1.4 \pm 0.42 \mathrm{mV} / \mathrm{s}$; BDNF, $5.02 \pm 0.41 \mathrm{mV} / \mathrm{s})$ at 5 weeks post-SCI $\left(F_{(6,21)}=10.47\right.$; Tukey's post-test; dorsal: blank vs BDNF, $p=0.04$; contralateral vs dorsal/blank, $p<0.0001$; contralateral vs dorsal/BDNF, $p=$
0.053; ANOVA; $n=4$ rats/group; Fig. $3 B, C$ ). No differences were noted in burst frequency (dorsal subregion: blank gel, $34.72 \pm$ 2.39 bursts/min; BDNF, $32 \pm 6.81$ bursts $/ \mathrm{min} ; F_{(5,14)}=1.05$; Tukey's post-test; ventral: blank vs BDNF, $p=0.9$; medial: blank vs BDNF, $p=0.92$; dorsal: blank vs BDNF, $p=0.9$; ANOVA; $n=$ 4 rats/group; Fig. $3 D$ ) or burst duration (dorsal subregion: blank gel, $0.50 \pm 0.06 \mathrm{~s} ;$ BDNF, $0.61 \pm 0.45 \mathrm{~s} ; F_{(5,14)}=1.87$, Tukey's post-test; ventral: blank vs BDNF, $p=0.59$; medial: blank vs BDNF, $p=0.90$; dorsal: blank vs BDNF, $p=0.62$; ANOVA; $n=$ 4 rats/group; Fig. $3 E$ ) between blank hydrogel and BDNF hydrogel groups at any diaphragm subregion, and these burst frequency and duration values in hydrogel-implanted contusion rats were similar to those in uninjured control animals that we assessed in previous work ( $\mathrm{Li}$ et al., 2015).

We also conducted CMAP recordings from the ipsilateral hemidiaphragm in response to supramaximal phrenic nerve stimulation. Unlike spontaneous EMG recordings, this technique allowed us to selectively quantify functional innervation of the diaphragm by PhMNs independent of the contribution of the descending bulbospinal drive. Unlike with EMGs in which we insert small needle electrodes into specific subregions of the muscle, we recorded CMAP amplitudes from the entire hemidiaphragm simultaneously using a surface electrode. We obtained CMAP recordings separately from both the ipsilateral and contralateral hemidiaphragm (Fig. 4A). Starting at 2 weeks postcontusion and continuing until the last recording time point at 3 weeks postcontusion, the BDNF hydrogel group (week 1, $5.70 \pm$ $0.28 \mathrm{mV}$; week 2, $6.33 \pm 0.48 \mathrm{mV}$; week 3, $6.56 \pm 0.31 \mathrm{mV}$ ) showed increased peak CMAP amplitude in the ipsilateral hemidiaphragm compared with the blank hydrogel group (week 1, 

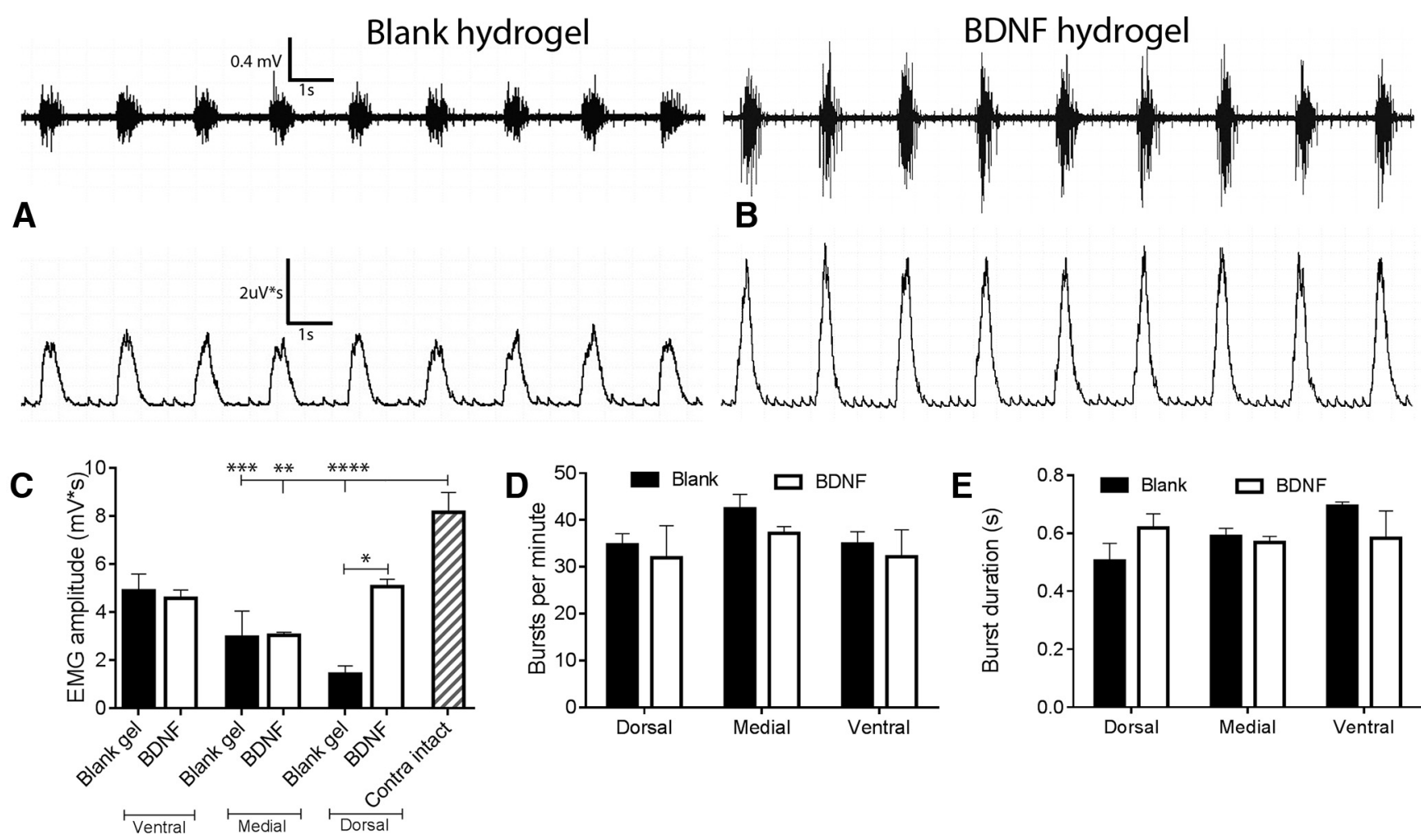

Figure 3. BDNF hydrogel implantation significantly improved diaphragm function following cervical contusion SCl. At 5 weeks after contusion/hydrogel implantation, we obtained EMG recordings from ipsilateral hemidiaphragm. $\boldsymbol{A}, \boldsymbol{B}$, Representative raw (top) and integrated (bottom) EMG traces for the blank hydrogel $(\boldsymbol{A})$ and BDNF hydrogel $(\boldsymbol{B})$ groups. $\boldsymbol{C}-\boldsymbol{E}$, We quantified integrated EMG amplitude $(\boldsymbol{C})$, burst frequency $(\boldsymbol{D})$, and burst duration $(\boldsymbol{E})$. We expressed results as the mean \pm SEM. ${ }^{*} p<0.05,{ }^{* *} p<0.01,{ }^{* * *} p<0.001,{ }^{* * * *} p<0.0001$.
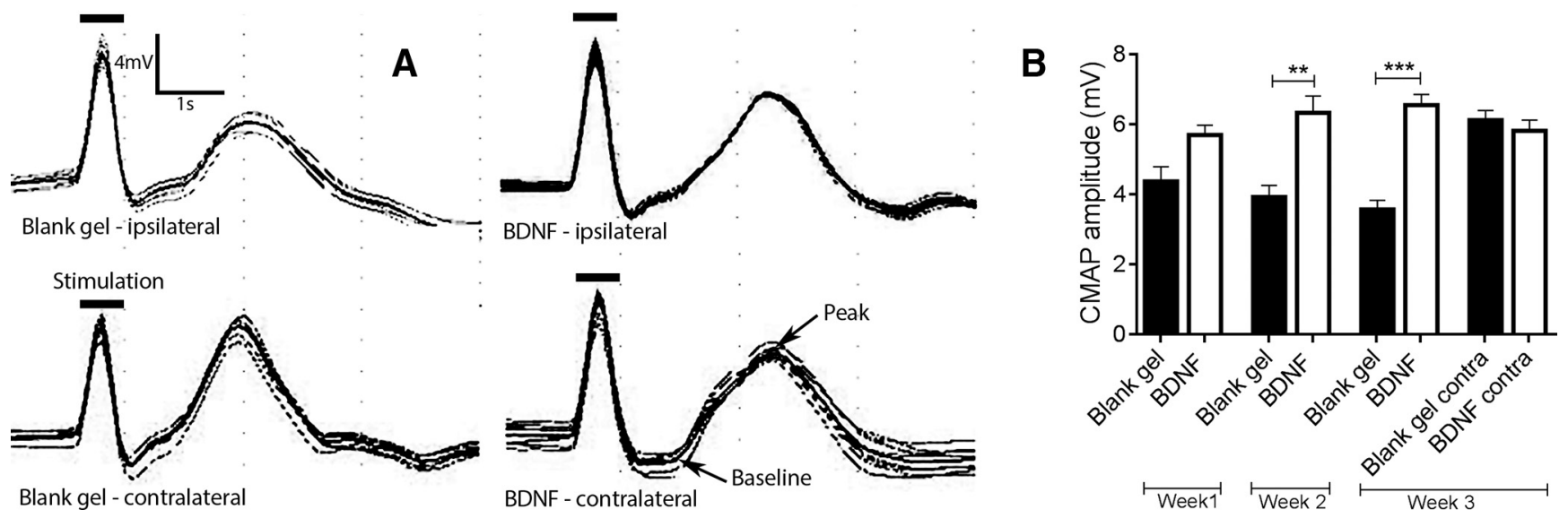

Figure 4. BDNF hydrogel implantation significantly improved functional diaphragm innervation following cervical contusion SCl. A, Following supramaximal phrenic nerve stimulation, we obtained CMAP recordings from ipsilateral and contralateral hemidiaphragm for both blank hydrogel (left traces) and BDNF hydrogel (right traces) groups. Representative traces are from 3 weeks postinjury. $\boldsymbol{B}$, We quantified CMAP amplitudes at different time points postinjury. We expressed results as the mean \pm SEM. ${ }^{* *} p<0.01,{ }^{* * *} p<0.001$.

$4.37 \pm 0.42 \mathrm{mV}$; week 2, $3.92 \pm 0.33 \mathrm{mV}$; week 3, 3.58 $\pm 0.27 \mathrm{mV}$; $F_{(7,24)}=11.64$, Tukey's post-test; blank vs BDNF: week $1, p=$ 0.15 ; week 2, $p=0.001$; week $3, p<0.0001$, ANOVA; $n=4$ rats/ group; Fig. 4B). Furthermore, CMAP amplitudes obtained from the ipsilateral hemidiaphragm of blank hydrogel animals were significantly reduced at all time points compared with the intact contralateral hemidiaphragm of the same animals (blank gel, $6.13 \pm 0.27$ $\mathrm{mV}$ ), while BDNF hydrogel rescued CMAP amplitudes in ipsilateral hemidiaphragm compared with the within-animal intact contralateral hemidiaphragm (BDNF, $5.82 \pm 0.3 \mathrm{mV}$; Fig. $4 B$ ).

Collectively, these electrophysiological findings using two powerful in vivo assessments of muscle activation demonstrate that implantation of BDNF hydrogel locally at the site of cervical contusion SCI robustly and persistently preserved diaphragm function.

BDNF hydrogel did not decrease lesion size, lesion volume, or cervical motor neuron cell body degeneration following cervical contusion SCI

To determine whether BDNF hydrogel improved diaphragmatic respiratory function after $\mathrm{C} 4 / \mathrm{C} 5$ contusion via a central neuroprotective effect within the cervical spinal cord, we quantified both lesion size and sparing of ventral horn motor neurons. In cresyl violet-stained transverse sections of the cervical spinal cord 

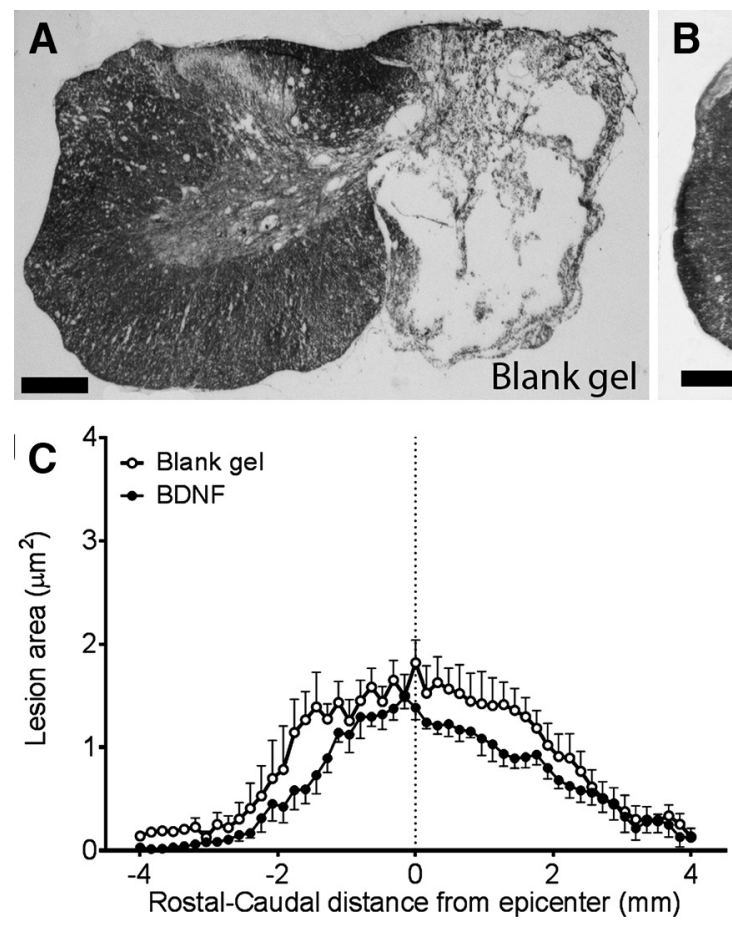

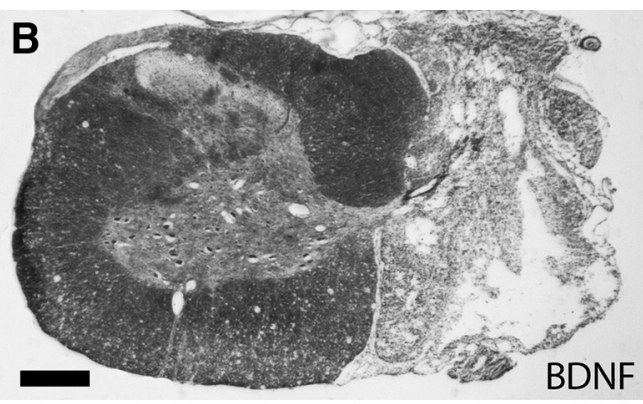

D

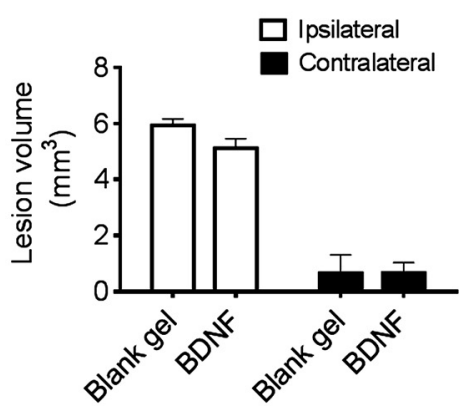

Figure 5. BDNF hydrogel did not decrease lesion size or lesion volume following cervical contusion $\mathrm{SCl}$. $\boldsymbol{A}-\boldsymbol{C}$, In cresyl violet-stained transverse sections of the cervical spinal cord at 5 weeks postcontusion, we found that BDNF hydrogel $(\boldsymbol{B})$ did not reduce lesion size compared with blank hydrogel $(\boldsymbol{A})$ at all rostral-caudal distances from the lesion epicenter ( $\boldsymbol{C}$. $\boldsymbol{D}$, BDNF hydrogel did not reduce overall lesion volume compared with blank hydrogel, as determined with the Cavalieri estimator of volume, in the hemicord both ipsilateral and contralateral to the contusion. Scale bar, 500 $\mu \mathrm{m}$. We expressed results as the mean \pm SEM.

at 5 weeks postcontusion, we found that BDNF hydrogel (Fig. 5B) did not reduce lesion size compared with blank hydrogel control (Fig. 5A) at all rostral-caudal distances from the lesion epicenter (Fig. $5 C ; p>0.05$ at all distances, ANOVA; $n=5$ /group). Furthermore, BDNF hydrogel did not reduce overall lesion volume compared with blank hydrogel-implanted animals, as determined with the Cavalieri estimator of volume (blank, $5.93 \pm 0.23$ $\mathrm{mm}^{3}$; BDNF, $5.11 \pm 0.34 \mathrm{~mm}^{3} ; F_{(3,16)}=44.42$, Tukey's post-test; $p=0.54$, ANOVA; $n=5$ rats/group; Figure $5 D)$. We also measured lesion size in the contralateral hemicord; we found littleto-no tissue damage in both groups, as well as no effect of BDNF on lesion volume compared with blank gel (blank, $0.65 \pm 0.64$ $\mathrm{mm}^{3}$; BDNF, $0.66 \pm 0.37 \mathrm{~mm}^{3}$, ANOVA; $n=5$ rats/group). In these same cresyl violet-stained sections from the cervical spinal cord, we found that BDNF hydrogel (Fig. 6B) also did not attenuate loss of ventral horn motor neurons located ipsilateral to the contusion compared with blank hydrogel control (Fig. 6A) at 5 weeks post-SCI. Specifically, we observed no effect of BDNF hydrogel on motor neuron counts that included all $\alpha$ motor neuron populations (not just PhMNs), both at defined rostral-caudal distances from the lesion epicenter $(p>0.05$ at all distances, ANOVA; $n=5$ /group; Fig. $6 C$ ) and across the entire cervical enlargement (blank gel, $4382 \pm 289$ motor neurons/animal; BDNF, $4603 \pm 362$ motor neurons/animal; $F_{(3,16)}=10.22$, Tukey's post-test; $p=0.90$, ANOVA; $n=5$ rats/group; Fig. $6 D$ ). BDNF also had no effects on motor neuron counts on the contralateral side (blank gel, $9724 \pm 827$ motor neurons/animal; BDNF, $10,406 \pm 788$ motor neurons/animal; $F_{(3,16)}=10.22$, Tukey's post-test; $p=0.22$, ANOVA; $n=5$ rats/group). Together, these histological findings suggest that the therapeutic mechanism of action of BDNF hydrogel was not central neuroprotection within the injured cervical spinal cord.
BDNF hydrogel significantly enhanced PhMN innervation of the diaphragm

Though BDNF hydrogel did not reduce secondary degeneration within the cervical spinal cord as assessed by lesion size and motor neuron somal counts, BDNF may have instead acted in a protective manner to maintain peripheral innervation at the diaphragm NMJ, the synapse that is critical for PhMN-diaphragm connectivity. To address this possibility, we selectively labeled $\mathrm{PhMN}$ cell bodies by intrapleurally injecting the retrograde axonal tracer, Alexa Fluor 647-conjugated CTB, into the ipsilateral hemidiaphragm. Importantly, we performed these CTB injections at 4 weeks postinjury/treatment to assess muscle innervation, because (1) PhMN soma will only be labeled with CTB following intrapleural injection if $\mathrm{NMJ}$ innervation is intact and (2) secondary PhMN loss is complete by this late injection time point (Nicaise et al., 2013). In sagittal sections of the spinal cord, CTB labeled a relatively compact column of PhMNs that extended from $\mathrm{C} 3$ to $\mathrm{C} 5$ (Fig. $7 A, B$ ). In transverse sections, we found CTB-labeled cells in the ipsilateral ventral horn at levels C3-C5 (Fig. 7C). Compared with uninjured rats (252.7 \pm 13.38 PhMNs), both blank hydrogel-implanted (61.67 $\pm 8.82 \mathrm{PhMNs})$ and BDNF hydrogel-implanted (156.7 $\pm 20.28 \mathrm{PhMNs})$ contusion animals showed reduced numbers of retrogradely labeled PhMNs $\left(F_{(2,6)}=40.96\right.$, Tukey's post-test; intact vs blank gel, $p=$ 0.004 ; intact vs BDNF, $p=0.0093$; ANOVA; $n=3$ rats/group). However, there were significantly greater numbers of CTBlabeled PhMNs in the C3-C5 spinal cord of BDNF hydrogel animals compared with blank hydrogel controls $\left(F_{(2,6)}=40.96\right.$, Tukey's post-test; $p=0.0097$, ANOVA; Fig. $7 D)$. Given the absence of motor neuron soma protection, these data suggest that BDNF hydrogel robustly preserved diaphragm innervation by PhMNs. 

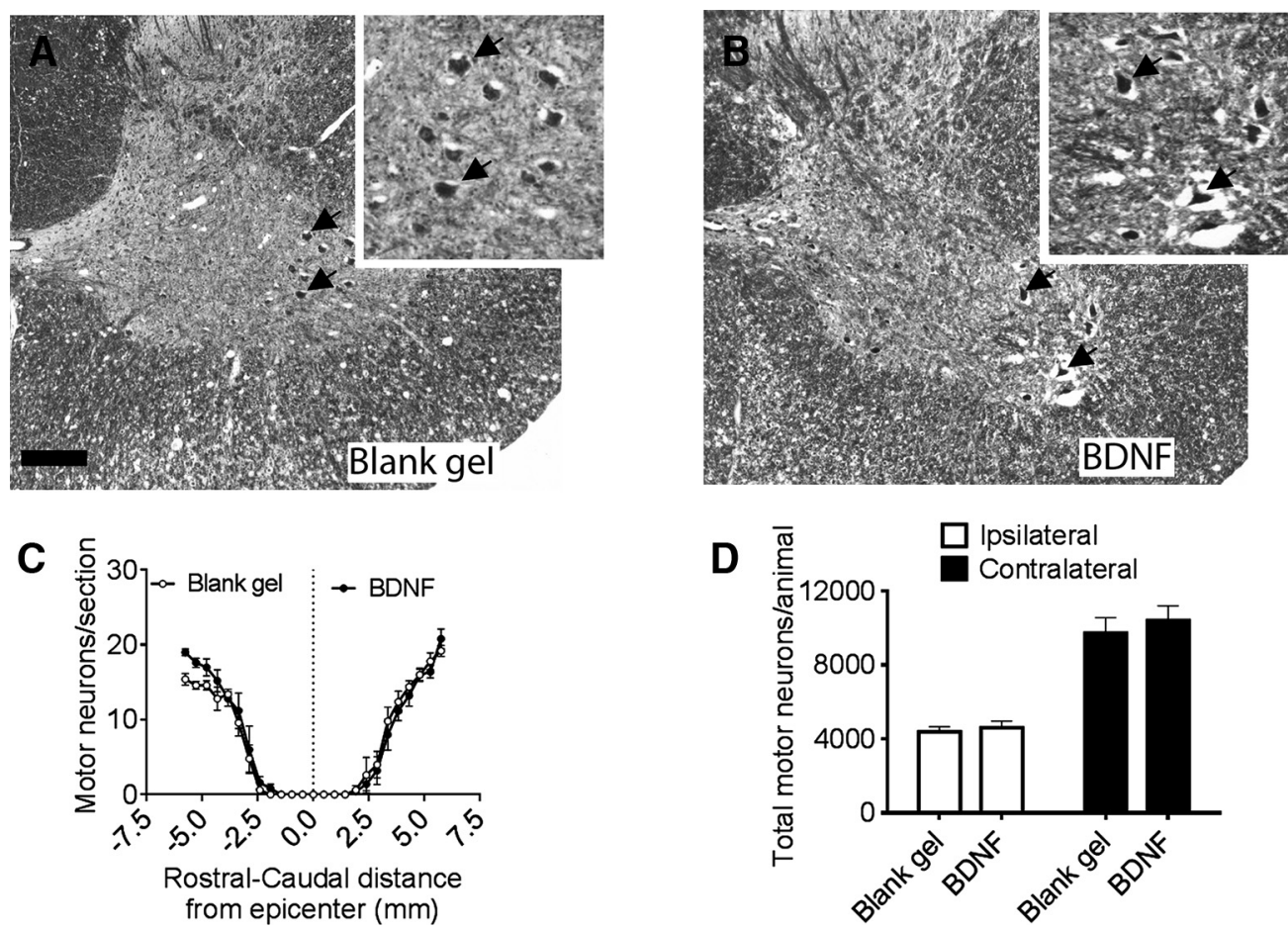

Figure 6. BDNF hydrogel did not decrease cervical motor neuron cell body degeneration following cervical contusion SCI. $\boldsymbol{A}, \boldsymbol{B}$, In cresyl violet-stained sections from the cervical spinal cord, we found that BDNF hydrogel $(B)$ did not attenuate the loss of ventral horn motor neurons located ipsilateral to the contusion compared with blank hydrogel $(\boldsymbol{A})$ at 5 weeks post-SCI (representative large motor neurons indicated by arrows). $\boldsymbol{C}, \boldsymbol{D}$, We observed no effect of BDNF hydrogel on total motor neuron counts, both at defined rostral-caudal distances from the lesion epicenter ( () and across the entire cervical enlargement in the hemicord both ipsilateral and contralateral to the contusion (D). We expressed results as the mean \pm SEM. Scale bar, $200 \mu \mathrm{m}$.
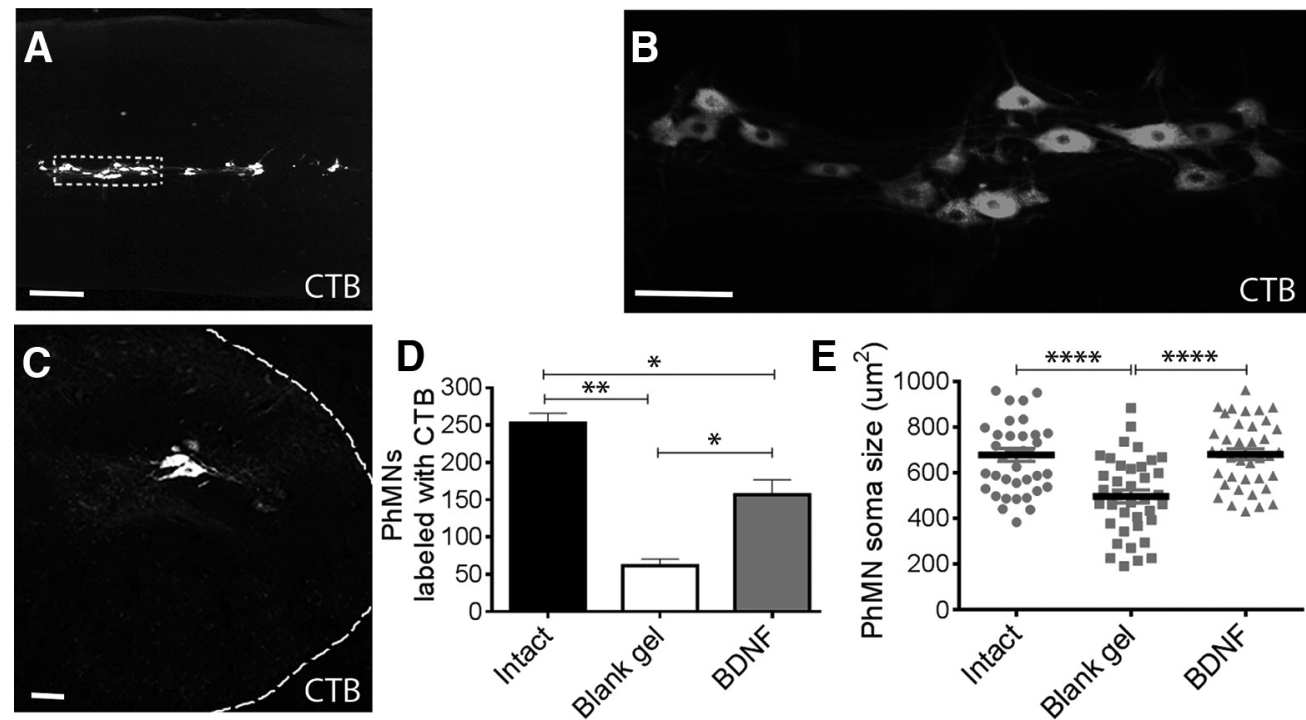

Figure 7. BDNF hydrogel increased counts of PhMNs retrogradely labeled from the diaphragm following cervical contusion SCI. We selectively labeled PhMN cell bodies by intrapleurally injecting the retrograde tracer Alexa Fluor 647-conjugated CTB into ipsilateral hemidiaphragm. $\boldsymbol{A}, \boldsymbol{B}$, In sagittal spinal cord sections, CTB labeled a relatively compact column of PhMNs that extended from C3 to C5. C, In transverse sections, we found CTB-labeled cells in ipsilateral ventral horn at levels C3-C5. D, There were significantly greater numbers of CTB-labeled PhMNs in the C3-C5 spinal cord of BDNF hydrogel animals compared with the blank hydrogel control. E, Somal size of CTB-labeled PhMNs in the BDNF hydrogel group was significantly greater than the blank hydrogel controls. Scale bars: $\boldsymbol{A}, 500 \mu \mathrm{m} ; \boldsymbol{B}, 100 \mu \mathrm{m} ; \boldsymbol{C}, 50 \mu \mathrm{m}$. We expressed results as the mean \pm SEM. ${ }^{*} p<0.05,{ }^{* *} p<0.01,{ }^{* * * *} p<0.0001$.

These findings show that BDNF delivered locally to the cervical spinal cord can exert important therapeutic effects on PhMNs spared the injury independent of overt somal protection. Along these lines, BDNF hydrogel implantation also significantly reduced cell body atrophy of CTB-labeled PhMNs (intact, 678.27 \pm $27.84 \mu \mathrm{m}^{2}$; blank gel, $496.47 \pm 27.74 \mu \mathrm{m}^{2}$; BDNF, $680.60 \pm$ $23.78 \mu \mathrm{m}^{2} ; F_{(2,111)}=15.84$, Tukey's post-test; intact vs blank gel, $p<0.0001$; blank gel vs BDNF, $p<0.0001$; intact vs BDNF, $p=$ 0.9 ; ANOVA; $n=38$ neurons/group; Fig. $7 E$ ), suggesting that BDNF may be able to improve the health of PhMNs after cervical SCI.

To more definitively assess the effects of BDNF hydrogel on diaphragm NMJ innervation, we performed detailed confocal morphological analysis of individual NMJ phenotypes in the 

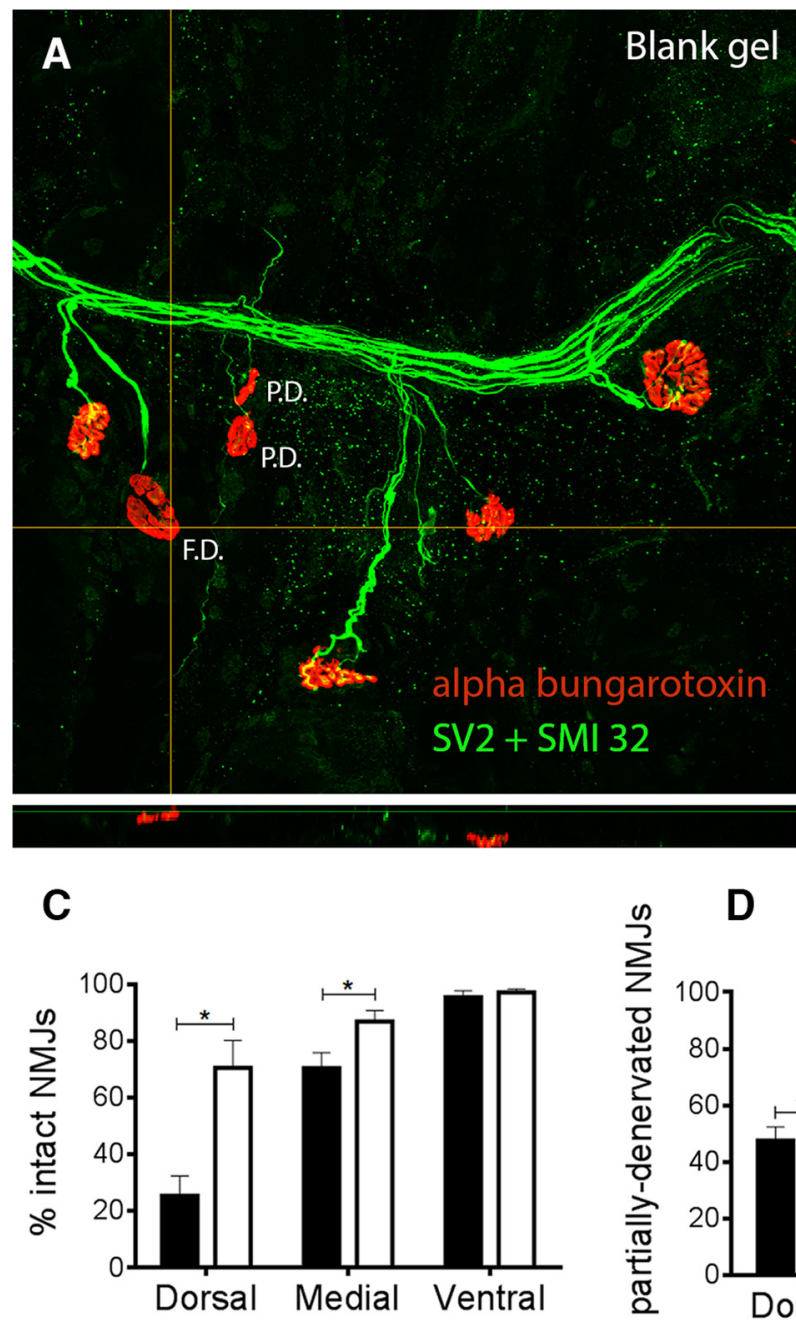

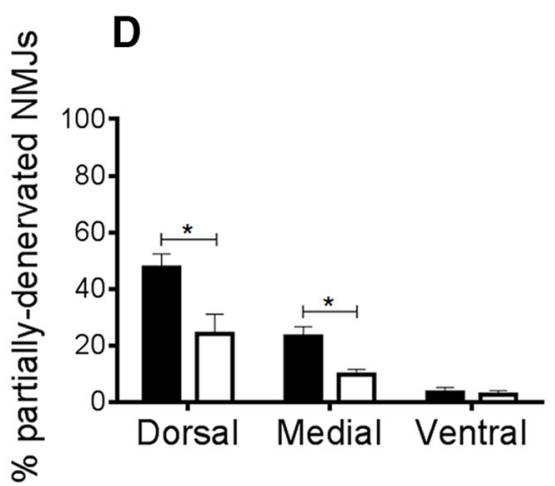

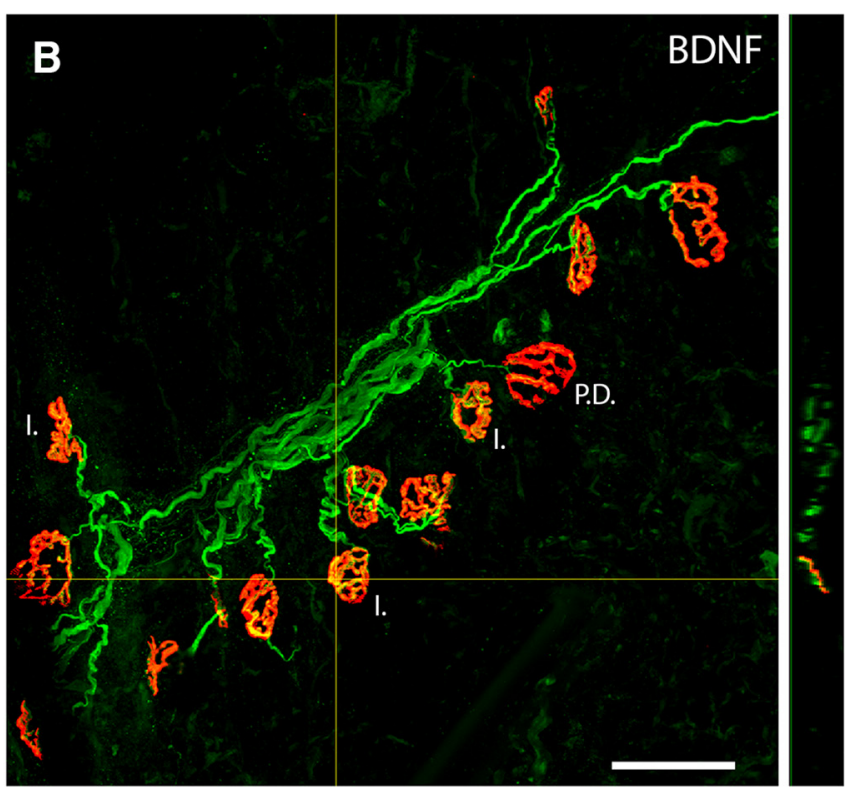
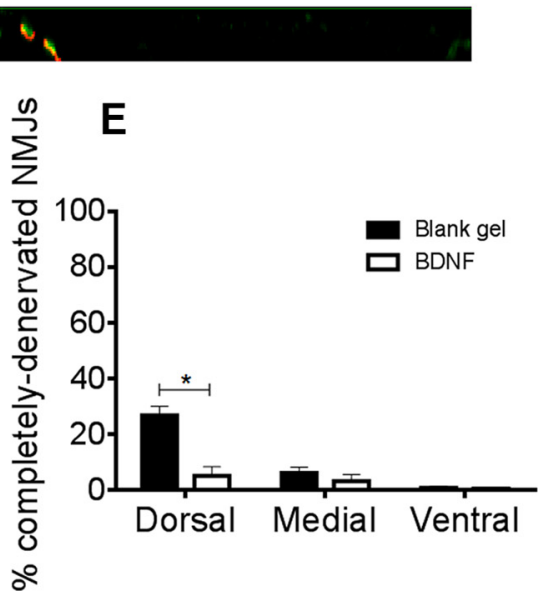

Figure 8. BDNF hydrogel preserved morphological innervation of the diaphragm by phrenic motor neurons following cervical contusion SCI. At 5 weeks postcontusion, we performed wholemount immunohistochemistry on hemidiaphragm by labeling phrenic motor axons all the way to their terminals with neurofilament (SMI-312) and SV2 antibodies. We also labeled postsynaptic nicotinic acetylcholine receptors in the muscle with Alexa Fluor 555-conjugated $\alpha$-bungarotoxin. $\boldsymbol{A}-\boldsymbol{E}$, Compared with blank hydrogel $(\boldsymbol{A})$, BDNF hydrogel $(\boldsymbol{B})$ significantly increased the percentage of fully innervated NMJs $(\boldsymbol{C})$, as well as decreased the percentage of both partially denervated $(\boldsymbol{D})$ and completely denervated $(\boldsymbol{E})$ NMJs. $(\boldsymbol{E}$, BDNF hydrogel implantation robustly preserved NMJ innervation at the dorsal subregion, but also exerted a more modest therapeutic effect on innervation at the medial subregion. We characterized individual NMJs as follows: intact (I.); fully denervated (F.D.); partially denervated (P.D.). Scale bar, $50 \mu \mathrm{m}$. We expressed results as the mean \pm SEM. ${ }^{*} p<0.05$.

hemidiaphragm ipsilateral to the contusion, including separately at dorsal, medial, and ventral subregions (Wright and Son, 2007; Wright et al., 2007, 2009). At 5 weeks postcontusion, we performed whole-mount immunohistochemistry on hemidiaphragm by labeling phrenic motor axons all the way to their terminals with neurofilament (SMI-312) and synaptic vesicle protein 2 (SV2) antibodies. We also labeled postsynaptic nicotinic acetylcholine receptors in the muscle with Alexa Fluor 555-conjugated $\alpha$-bungarotoxin. Compared with blank hydrogel (Fig. $8 A$ ), BDNF hydrogel (Fig. $8 B$ ) significantly increased the percentage of fully innervated NMJs in the dorsal and medial subregions of the hemidiaphragm (dorsal: blank $=25.4 \pm 7.01 \%$; $\mathrm{BDNF}=$ $70.65 \pm 9.6 \% ; p=0.0003$; medial: blank $=70.47 \pm 4.83 \%$; $\mathrm{BDNF}=86.95 \pm 3.92 \% ; p=0.035$; ventral: blank $=95.57 \pm$ $2.42 \% ; \mathrm{BDNF}=97.17 \pm 1.23 \% ; F_{(5,18)}=22.05$, Tukey's post-test ANOVA; $n=4$ rats/group; Fig. $8 C$ ). In addition, compared with blank hydrogel, BDNF decreased the percentage of both partially denervated NMJs (dorsal: blank $=47.65 \pm 4.83 \%$; $\mathrm{BDNF}=$ $24.17 \pm 7.04 \% ; p=0.0058$; medial: blank $=23.27 \pm 3.4 \%$;
$\mathrm{BDNF}=9.77 \pm 1.93 \% ; p=0.02$; ventral: blank $=3.57 \pm 2.03 \%$, $\mathrm{BDNF}=2.82 \pm 1.34 \% ; F_{(5,18)}=18.75$, Tukey's post-test, ANOVA; $n=4$ rats/group; Fig. $8 D$ ) and completely denervated NMJs (dorsal: blank $=26.9 \pm 3.18 \% ; \mathrm{BDNF}=5.2 \pm 3.22 \% ; p<$ 0.0001 ; medial: blank $=6.25 \pm 2.05 \%$; $\mathrm{BDNF}=3.27 \pm 2.35 \%$; $p=0.93$; ventral: blank $=0.90 \pm 0.54 \% ; \mathrm{BDNF}=0.50 \pm 0.28 \%$; $F_{(5,18)}=19.21$; Tukey's post-test, ANOVA; $n=4$ rats/group; Fig. $8 E$ ). In agreement with the EMG recordings (Fig. $3 C$ ), we observed little diaphragm denervation in the ventral subregion of the muscle, while the most significant denervation occurred in the dorsal subregion and a smaller but still significant amount of denervation was noted in the medial subregion (Fig. $8 C-E$ ). Just as with the preservation of EMG amplitudes seen in the dorsal subregion, BDNF hydrogel implantation robustly enhanced NMJ innervation at the dorsal subregion, but also exerted a more modest therapeutic effect on innervation at the medial subregion. We previously showed that the contralateral hemidiaphragm is not significantly affected in this unilateral cervical contusion model (Nicaise et al., 2012); therefore, we have 
not conducted this analysis of contralateral muscle again in the current study.

Collectively, these findings with retrograde labeling and NMJ morphology analysis (along with CMAPs) demonstrate for the first time that BDNF significantly enhanced diaphragm NMJ innervation by PhMNs after SCI.

\section{BDNF hydrogel implantation following cervical contusion enhanced serotonergic innervation of PhMNs}

BDNF may have also exerted therapeutic effects on respiratory neural circuitry via mechanisms in addition to enhanced NMJ innervation. Descending serotonergic input plays an important role in regulating the excitability of spinal cord motor neurons, in particular their response to glutamatergic signaling (Perrier et al., 2013). It has been reported that BDNF-expressing mesenchymal stem cells significantly increased 5-HT fiber growth after SCI (Sasaki et al., 2009). Therefore, in the context of diaphragm function, BDNF may be able to strengthen rhythmic excitatory input to PhMNs from spared and/or regrowing rVRG axons by increasing serotonergic innervation of PhMNs via 5-HT fiber sprouting. To address this possibility, we quantified 5-HT immunostaining in the $\mathrm{C} 3$-C5 ventral horn ipsilateral to the contusion at 5 weeks postcontusion. Compared with blank hydrogel control (Fig. $9 A, C$ ), we found that BDNF hydrogel (Fig. $9 B, D$ ) enhanced the density of serotonergic axons directly surrounding CTB-labeled PhMNs.

We extended this analysis of 5-HT fiber growth within the PhMN pool by performing quantification of (1) numbers of $5-\mathrm{HT}^{+}$axon profiles, (2) length of these $5-\mathrm{HT}^{+}$axon profiles, and (3) integrated intensity of 5-HT immunostaining at multiple defined distances from CTB-labeled PhMNs in transverse sections (Fig. 9E,F). We present each of these datasets at three separate distances from PhMNs to show the proximity effects of BDNF hydrogel on 5-HT axonal growth responses with respect to the location of PhMNs. Compared with blank hydrogel control, $\mathrm{BDNF}$ increased the number of $5-\mathrm{HT}^{+}$axonal profiles at all three distances $\left(F_{(5,30)}=27.55\right.$, Tukey's post-test; $0-50 \mu \mathrm{m}$ : blank $=$ $49.83 \pm 5.84 ; \mathrm{BDNF}=100 \pm 7.35 ; p=0.04 ; 50-100 \mu \mathrm{m}:$ blank $=111.6 \pm 6.0 ; \mathrm{BDNF}=162.50 \pm 7.61 ; p=0.03 ; 100-150$ $\mu \mathrm{m}$ : blank $=135.83 \pm 10.68 ; \mathrm{BDNF}=223.33 \pm 21.55 ; p<$ 0.0001 , ANOVA; $n=3$ rats/group; Fig. $9 G)$. BDNF also increased total $5-\mathrm{HT}^{+}$axonal length at all three distances $\left(F_{(5,30)}=76.80\right.$, Tukey's post-test; $0-50 \mu \mathrm{m}$ : blank $=272.72 \pm 20.01 \mu \mathrm{m}$; $\mathrm{BDNF}=712.17 \pm 37.91 ; p<0.0001 ; 50-100 \mu \mathrm{m}$ : blank $=$ $776.47 \pm 63.94 ; \mathrm{BDNF}=1253.12 \pm 34.54 ; p<0.0001 ; 100-150$ $\mu \mathrm{m}$ : blank $=901.11 \pm 40.24 ; \mathrm{BDNF}=1565.89 \pm 84.01 ; p<$ 0.0001 , ANOVA; $n=3$ rats/group; Fig. $9 H$ ). In addition, BDNF increased integrated intensity of 5-HT immunostaining compared with blank hydrogel $\left(F_{(5,30)}=78.08\right.$, Tukey's post-test; $0-50 \mu \mathrm{m}$ : blank $=0.74 \pm 0.08$ a.u.; $\mathrm{BDNF}=1.91 \pm 0.10$ a.u.; $p=0.04 ; 50-100 \mu \mathrm{m}$ : blank $=3.49 \pm 0.14$ a.u.; $\mathrm{BDNF}=4.88 \pm$ 0.29 a.u.; $p=0.009 ; 100-150 \mu \mathrm{m}$ : blank $=5.16 \pm 0.23$ a.u.; $\mathrm{BDNF}=7.14 \pm 0.49$ a.u.; $p=0.0001, \mathrm{ANOVA} ; n=3$ rats/group; Fig. 9I).

Last, we conducted quantitative analysis of synaptic input to PhMNs by serotonergic axons using multilabeling immunohistochemistry for $5-\mathrm{HT}^{+}$axons, $\mathrm{CTB}^{+}$postsynaptic PhMNs, and the presynaptic marker synaptophysin. We assessed the number of putative synaptic connections between 5-HT fibers and CTBlabeled PhMNs using confocal acquisition of $z$-stacks and quantification of 5-HT axon-PhMN contacts using single- $z$ section analysis to establish direct apposition of presynaptic $5-\mathrm{HT}^{+} /$synaptophysin ${ }^{+}$axon terminals and postsynaptic $\mathrm{CTB}^{+}$PhMNs.
Compared with blank hydrogel control (Fig. 10A) and BDNF hydrogel (Fig. 10B) animals showed significantly more putative synaptic contacts between 5-HT axons and PhMNs (blank gel, $1.6 \pm 0.29 / \mathrm{PhMN}$ soma; BDNF, $5.2 \pm 0.35 ; t=7.86$, $\mathrm{df}=28 ; p<$ 0.0001 , unpaired $t$ test; $n=4$ rats/group; Fig. 10C).

Collectively, these results show that BDNF hydrogel promoted plasticity of axonal populations associated with enhanced $\mathrm{PhMN}$ excitability. This represents an additional mechanism potentially responsible for functional recovery, particularly the increased EMG amplitudes that may be associated with rhythmic drive to PhMNs from supraspinal respiratory centers.

\section{Discussion}

\section{Novel biomaterial-based strategy for SCI repair}

As a viable drug delivery system for BDNF therapeutics, we have developed a hydrogel loaded with BDNF. All of the materials used in this delivery platform are biocompatible natural polysaccharides that have been shown to be safe for human use (Komai et al., 2005; Scarano et al., 2009; Neimert-Andersson et al., 2014). The BDNF-loaded hydrogel can be implanted subdurally for local BDNF delivery at the injury site without damaging spinal cord tissue. Clinically, the hydrogel could be implanted, for example, when spine decompression/stabilization surgery is performed so that no additional surgery will be required for hydrogel administration.

Our innovative approach is significant for developing a therapy for respiratory dysfunction after SCI because we are harnessing the power of biomaterial design to address both biological considerations (i.e., the delivery of specific therapeutic factors to target relevant pathogenic mechanisms) and practical issues (i.e., local delivery to the injury site with appropriate dosing and duration). We found that BDNF exerted effects on peripheral mechanisms such as enhanced NMJ innervation and central reparative processes such as stimulating 5-HT axonal growth.

Our BDNF hydrogel releases relatively high amounts of BDNF in the first $2 \mathrm{~d}$, followed by low-dose release (364.2-29.5 ng/d) for $>17 \mathrm{~d}$. While the formulation we assessed was functionally and histopathologically efficacious, the optimal dose and duration of BDNF treatment for cervical SCI remain unknown. Furthermore, persistently high levels of BDNF within the spinal cord can result in unwanted side effects such as neuropathic pain, hyperreflexia, and abnormal motor function (Boyce and Mendell, 2014). Therefore, it will be important in future work to test additional release profiles to identify the most effective treatment paradigm with minimized side effects.

\section{Local delivery of BDNF hydrogel is a clinically viable approach for reducing secondary damage to respiratory circuitry following SCI}

Despite a body of work showing the neuroprotective effects of BDNF after axotomy, in SCI models, and in degenerative diseases such as ALS (Weishaupt et al., 2012), our data do not show protective effects on cervical motor neuron survival or lesion size. Instead, our results shed light on an incredibly important outcome of secondary injury that has not received extensive attention: peripheral muscle denervation by the axon terminals of motor neurons spared by the injury. Even though the cell bodies of these neurons persist, the circuit is incomplete. BDNF signaling through TrkB normally acts to maintain diaphragm NMJ structure and PhMN innervation in intact/non-SCI conditions (Mantilla et al., 2014b; Greising et al., 2015); BDNF hydrogel delivery in our model may represent an extension of this important function in the injured setting. These data with BDNF hydrogel implantation demonstrate the therapeutic 

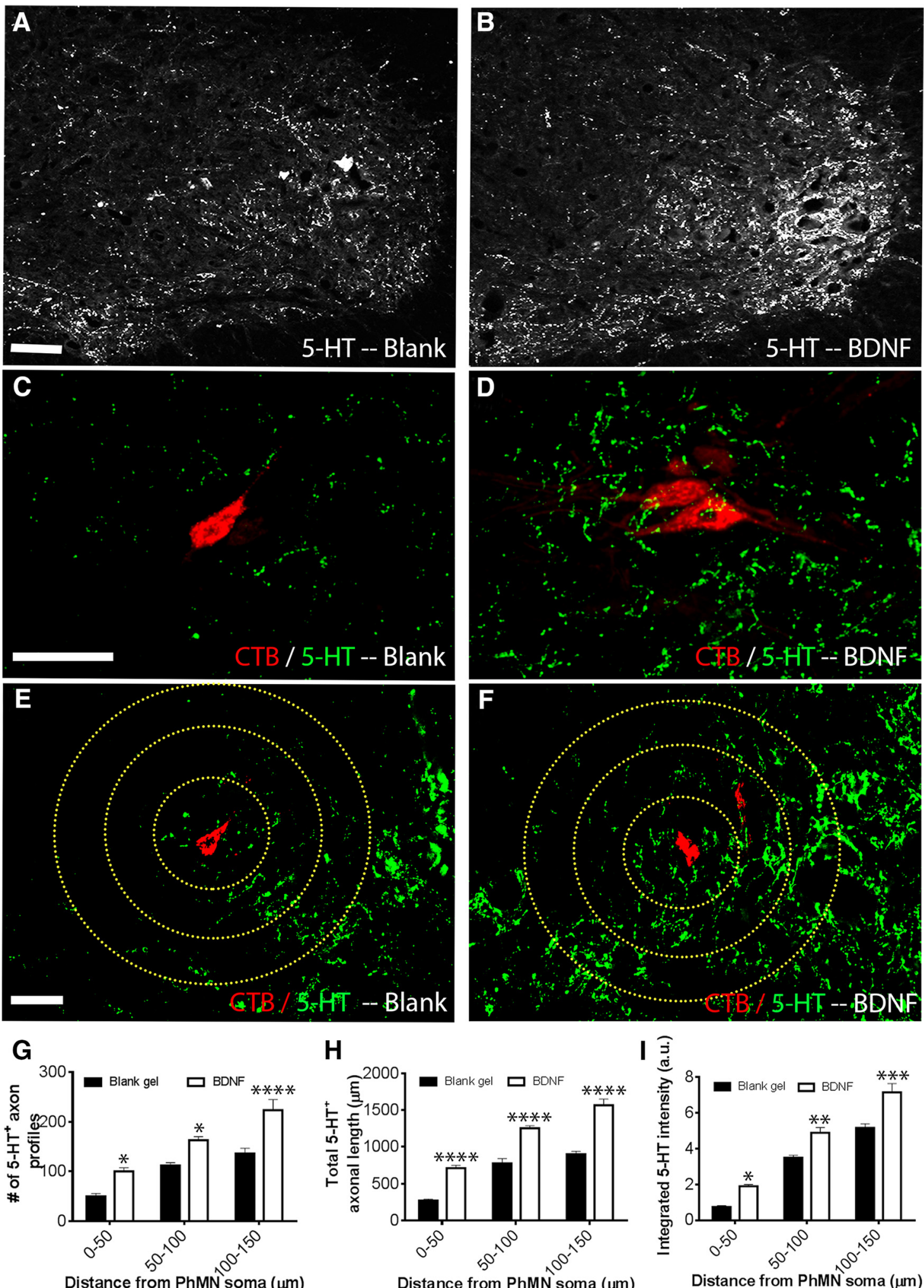

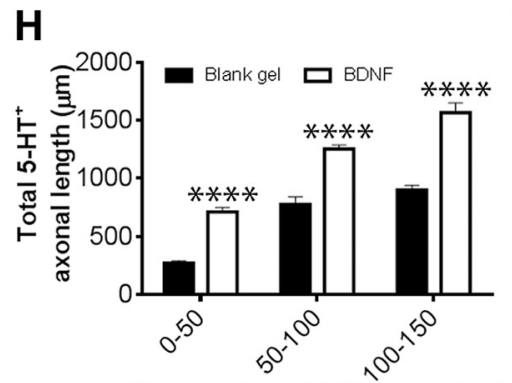

Distance from PhMN soma $(\mu \mathrm{m})$

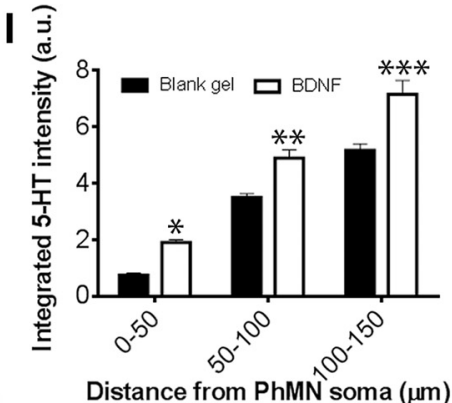

Distance from PhMN soma $(\mu \mathrm{m})$

Figure 9. BDNF hydrogel implantation following cervical contusion enhanced serotonergic innervation of PhMNs. We quantified 5-HT immunostaining in the C3-C5 ventral horn ipsilateral to the contusion at 5 weeks postinjury. $\boldsymbol{A}-\boldsymbol{D}$, Compared with blank hydrogel control $(\boldsymbol{A}, \boldsymbol{C})$, BDNF hydrogel $(\boldsymbol{B}, \boldsymbol{D})$ enhanced the density of serotonergic axons surrounding $(\mathrm{TB}$-labeled PhMNs at 5 weeks postcontusion. $\boldsymbol{E}-\mathbf{I}$, Compared with blank hydrogel control $(\boldsymbol{E}), \mathrm{BDNF}(\boldsymbol{F})$ increased the number of $5-\mathrm{HT}^{+}$axonal profiles $(\boldsymbol{G})$, total $5-\mathrm{HT}^{+}{ }^{+}$axonal length $(\boldsymbol{H})$, and integrated intensity of 5 -HT immunostaining $(I)$ at multiple defined distances from CTB-labeled PhMNs. Scale bars: $\boldsymbol{A}, \boldsymbol{B}, \boldsymbol{E}, \boldsymbol{F}, 50 \mu \mathrm{m} ; \boldsymbol{C}, \boldsymbol{D}, 100 \mu \mathrm{m}$. We expressed results as the mean $\pm S E M .{ }^{*} p<0.05,{ }^{* *} p<0.01,{ }^{* * *} p<$ $0.0011^{* * * *} p<0.0001$. 

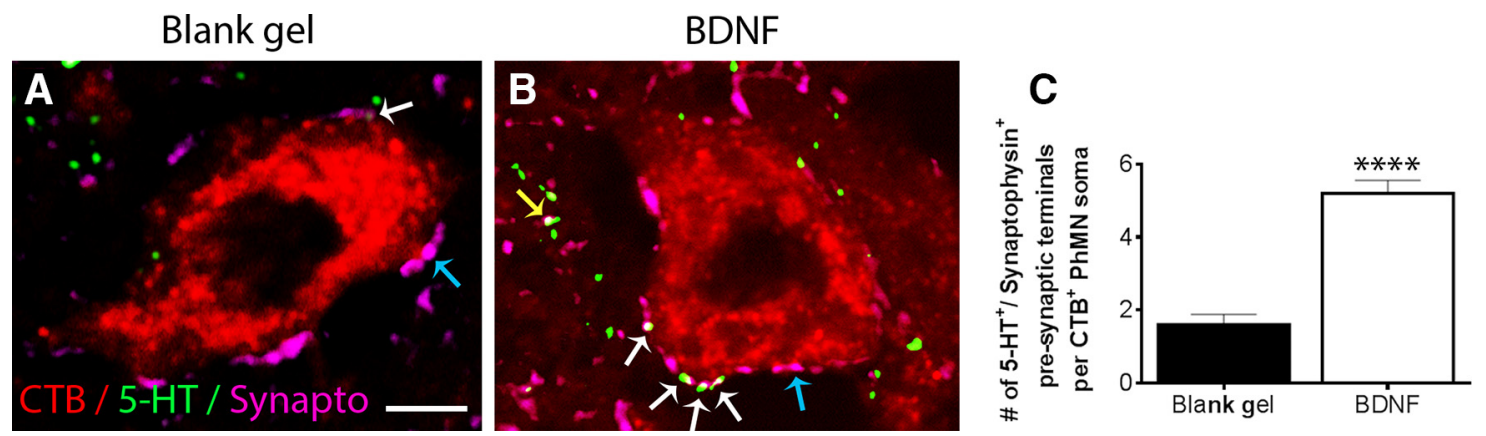

Figure 10. BDNF hydrogel implantation increased $5-\mathrm{HT}^{+}$/synaptophysin ${ }^{+}$presynaptic terminals at $\mathrm{CTB}^{+} \mathrm{PhMN}$ soma. We assessed the number of putative synaptic connections between 5 -HT fibers and CTB-labeled PhMNs using confocal acquisition of z-stacks and quantification of 5-HT axon-PhMN contacts using single-z section analysis to establish the direct apposition of presynaptic 5 - $\mathrm{HT}^{+}$/synaptophysin ${ }^{+}$axon terminals and postsynaptic CTB ${ }^{+}$PhMNs. $\boldsymbol{A}-\boldsymbol{C}$, Compared with blank hydrogel control $(\boldsymbol{A})$, BDNF hydrogel $(\boldsymbol{B})$ animals showed significantly more putative synaptic contacts between $5-\mathrm{HT}$ axons and PhMNs (C). We expressed results as the mean \pm SEM. Scale bar, $10 \mu \mathrm{m}$. ${ }^{* * * *} p<0.0001$.

power of addressing muscle denervation that underlies persistent diaphragm paralysis.

Our NMJ morphology data are only from a single late time point, raising the possibility that, instead of acting via initial preservation of NMJ innervation, BDNF hydrogel promoted NMJ reinnervation following initial denervation (or a combination of both). It is challenging to distinguish between an NMJ that was never denervated and one that was denervated and subsequently reinnervated as reinnervated NMJs often appear morphologically normal by this late time point postinsult. However, we observed CMAP preservation starting at the relatively early time of 2 weeks post-SCI, supporting the contention that BDNF hydrogel was acting to prevent initial denervation at diaphragm NMJ. Furthermore, we did not observe any hallmarks of peripheral plasticity at the NMJ with BDNF hydrogel such as terminal sprouting, nodal sprouting, or regrowth of initially retracted phrenic motor axons, though we also performed this assessment at a late time point long after BDNF stopped being released from the hydrogel. On the other hand, although the effects of BDNF hydrogel on 5-HT axonal growth around the CTB-labeled PhMNs could be due to initial preservation instead of sprouting, we observed increased 5-HT fiber density with BDNF hydrogel rostral to the contusion at locations where secondary degeneration is not significant, suggesting that BDNF is promoting the growth of these axons and not sparing.

\section{BDNF hydrogel may also be acting via various reparative mechanisms}

Given the clinical relevance of delayed intervention in the SCI patient population, it will be important in future work to test hydrogel implantation at time points beyond the window of early secondary degeneration. Reparative effects of interventions such as BDNF delivery may be viable targets in this context, including the sprouting of spared motor axons and reinnervation of denervated endplates that is a potentially powerful substrate for promoting functional recovery. To this point, $\mathrm{NMJ}$ reinnervation is a mechanism by which functional recovery can occur after motor neuron injury, including spontaneously without therapeutic interventions. In our cervical contusion model, we previously demonstrated that partial loss of the PhMN pool is followed by sprouting and reinnervation of a portion of the denervated junctions, which was associated with progressive, spontaneous recovery of diaphragm function as assessed by CMAP amplitudes (though recovery was only minimal; Nicaise et al., 2013). BDNF hydrogel implantation may enhance this process of NMJ reinnervation. In addition to our data demonstrating morphological effects at the NMJ, previous work has also shown that BDNF can induce other changes at the diaphragm NMJ. For example, BDNF enhances synaptic transmission at the diaphragm NMJ (Mantilla et al., 2004), including in the $\mathrm{C} 2$ hemisection model using intrapleural adeno-associated virus-BDNF injection (Gransee et al., 2013), suggesting additional peripheral mechanisms relevant to diaphragm recovery.

Other reparative mechanisms may also be influenced by BDNF delivered locally to the injured cervical spinal cord. For example, BDNF hydrogel may be capable of modulating PhMN properties such as intrinsic excitability and response to bulbospinal input (Gill et al., 2016). This strengthened synaptic input to PhMNs could be from the small number of ipsilateral rVRG axons spared by contusion and/or latent contralateral rVRG input that is mostly inactive before injury. BDNF can increase the excitability of spinal cord motor neurons involved in the control of limb function (Boyce et al., 2012; Boyce and Mendell, 2014). The relatively small amount of spontaneous diaphragm recovery that occurs after $\mathrm{C} 2$ hemisection SCI is mediated in part by BDNF acting upon TrkB receptors expressed by PhMNs (Mantilla et al., 2013, 2014a). Diaphragm activation by PhMNs can also be enhanced in C2 hemisection with intrathecal BDNF injection (Mantilla et al., 2013), intraspinal transplantation of mesenchymal stem cells engineered to overexpress BDNF (Gransee et al., 2015), and selective overexpression of TrkB in PhMNs (Gransee et al., 2013; Martínez-Gálvez et al., 2016). Using phrenic nerve recordings as a measure of PhMN output, BDNF has also been shown to play a key role in the plasticity of spared glutamatergic input to PhMNs that underlies the partial recovery of diaphragm function observed after cervical hemisection (Dale-Nagle et al., 2010). Given this body of work, it will be important moving forward to test the effects of our BDNF hydrogel on PhMN excitability, particularly at the single-cell level and in response to bulbospinal synaptic input.

We found that BDNF hydrogel did not improve EMG amplitude in the ventral and medial subregions of the hemidiaphragm, but only in the dorsal portion of the muscle. We hypothesize that the beneficial effect of BDNF hydrogel on EMG burst amplitudes in the dorsal hemidiaphragm may be due to the location of hydrogel implantation. Based on previous work from both our laboratory and others, it has been shown that different subregions of the hemidiaphragm are innervated by phrenic motor neurons located at different locations within the C3-C5 spinal cord (Li et al., 2015). In general, PhMNs whose soma are found at C3 primarily innervate the ventral portion of the muscle. PhMNs at the opposite end of the PhMN pool (C5) primarily innervate the 
dorsal hemidiaphragm subregion, while those at $\mathrm{C} 4$ primarily innervate the medial part of the muscle. Our unilateral contusion injury is located at the C4/C5 level, which explains why the EMG burst amplitudes in blank-hydrogel control animals are lowest at the dorsal and medial subregions (particularly the dorsal subregion). We implanted the BDNF hydrogel (as well as the control blank hydrogel) directly above the contusion epicenter; therefore, we hypothesize that the local delivery of BDNF exerted effects close to the hydrogel implantation site, which may explain the selective effects in the dorsal hemidiaphragm.

\section{BDNF hydrogel promoted serotonergic axon growth}

BDNF acting through TrkB plays an important role in neuroplasticity following SCI, including the regeneration of axotomized neurons and the sprouting of spared fibers (Weishaupt et al., 2012). A number of previous studies has shown that BDNF treatment in SCI models increases the survival of neurons and axonal growth when delivered by intrathecal infusion (Bregman et al., 1997; Ye and Houle, 1997; Novikova et al., 2000, 2002), intraspinal viral transduction (Koda et al., 2004; Boyce et al., 2012), and stem/progenitor cell transplantation (Lu et al., 2005; Lynskey et al., 2006). Along these lines, we observed robustly increased PhMN innervation by 5 -HT axons in response to BDNF hydrogel, presumably via an axonal growth response (and not via sparing, as discussed above). BDNF hydrogel may have also restored diaphragm function in our cervical contusion model by promoting the regrowth of injured bulbospinal rVRG axons and synaptic reconnection with spared PhMNs. Furthermore, the generation of novel connections (that differ from the original preinjury circuitry) by growing axons can promote some degree of functional recovery post-SCI, either spontaneously (Bareyre et al., 2004) or in response to the manipulation of axonal growth inhibitors (Siegel et al., 2015). Accordingly, BDNF hydrogel may have resulted in reinnervation of PhMNs by sprouting contralateral rVRG axons spared by the injury. Supporting these possibilities, bulbospinal axon populations have repeatedly shown axon growth responses to BDNF (Weishaupt et al., 2012).

\section{Conclusions}

Our findings may have profound implications for SCI patients with persistent diaphragm dysfunction. We have developed a novel and safe biomaterial-based platform to locally deliver BDNF to the injured cervical spinal cord with controlled dosing and duration. Furthermore, in a clinically relevant animal model of cervical contusion SCI, we have successfully demonstrated the therapeutic efficacy of this promising drug delivery system for effectively repairing PhMN-diaphragm circuitry and maintaining diaphragmatic respiratory function. In addition, we demonstrate novel mechanisms by which local BDNF delivery to an SCI site can repair respiratory neural circuitry simultaneously at multiple neuroanatomical locations: enhanced diaphragm innervation peripherally at the NMJ and increased PhMN innervation by descending 5-HT axons centrally within the ventral horn of the cervical spinal cord.

\section{References}

Bareyre FM, Kerschensteiner M, Raineteau O, Mettenleiter TC, Weinmann O, Schwab ME (2004) The injured spinal cord spontaneously forms a new intraspinal circuit in adult rats. Nat Neurosci 7:269-277. CrossRef Medline

Boyce VS, Mendell LM (2014) Neurotrophins and spinal circuit function. Front Neural Circuits 8:59. CrossRef Medline

Boyce VS, Park J, Gage FH, Mendell LM (2012) Differential effects of brain- derived neurotrophic factor and neurotrophin-3 on hindlimb function in paraplegic rats. Eur J Neurosci 35:221-232. CrossRef Medline

Bregman BS, McAtee M, Dai HN, Kuhn PL (1997) Neurotrophic factors increase axonal growth after spinal cord injury and transplantation in the adult rat. Exp Neurol 148:475-494. CrossRef Medline

Dale-Nagle EA, Hoffman MS, MacFarlane PM, Satriotomo I, Lovett-Barr MR, Vinit S, Mitchell GS (2010) Spinal plasticity following intermittent hypoxia: implications for spinal injury. Ann NY Acad Sci 1198:252-259. CrossRef Medline

Gill LC, Gransee HM, Sieck GC, Mantilla CB (2016) Functional recovery after cervical spinal cord injury: role of neurotrophin and glutamatergic signaling in phrenic motoneurons. Respir Physiol Neurobiol 226:128136. CrossRef Medline

Goswami DB, May WL, Stockmeier CA, Austin MC (2010) Transcriptional expression of serotonergic regulators in laser-captured microdissected dorsal raphe neurons of subjects with major depressive disorder: sexspecific differences. J Neurochem 112:397-409. CrossRef Medline

Gransee HM, Zhan WZ, Sieck GC, Mantilla CB (2013) Targeted delivery of TrkB receptor to phrenic motoneurons enhances functional recovery of rhythmic phrenic activity after cervical spinal hemisection. PLoS One 8:e64755. CrossRef Medline

Gransee HM, Zhan WZ, Sieck GC, Mantilla CB (2015) Localized delivery of brain-derived neurotrophic factor-expressing mesenchymal stem cells enhances functional recovery following cervical spinal cord injury. J Neurotrauma 32:185-193. CrossRef Medline

Greising SM, Stowe JM, Sieck GC, Mantilla CB (2015) Role of TrkB kinase activity in aging diaphragm neuromuscular junctions. Exp Gerontol 72: 184-191. CrossRef Medline

Hernandez-Torres V, Gransee HM, Mantilla CB, Wang Y, Zhan WZ, Sieck GC (2017) BDNF effects on functional recovery across motor behaviors after cervical spinal cord injury. J Neurophysiol 117:537-544. CrossRef Medline

Issa AN, Zhan WZ, Sieck GC, Mantilla CB (2010) Neuregulin-1 at synapses on phrenic motoneurons. J Comp Neurol 518:4213-4225. CrossRef Medline

Kinkead R, Zhan WZ, Prakash YS, Bach KB, Sieck GC, Mitchell GS (1998) Cervical dorsal rhizotomy enhances serotonergic innervation of phrenic motoneurons and serotonin-dependent long-term facilitation of respiratory motor output in rats. J Neurosci 18:8436-8443. CrossRef Medline

Koda M, Hashimoto M, Murakami M, Yoshinaga K, Ikeda O, Yamazaki M, Koshizuka S, Kamada T, Moriya H, Shirasawa H, Sakao S, Ino H (2004) Adenovirus vector-mediated in vivo gene transfer of brain-derived neurotrophic factor (BDNF) promotes rubrospinal axonal regeneration and functional recovery after complete transection of the adult rat spinal cord. J Neurotrauma 21:329-337. CrossRef Medline

Komai H, Naito Y, Okamura Y (2005) Dextran sulfate as a leukocyteendothelium adhesion molecule inhibitor of lung injury in pediatric open-heart surgery. Perfusion 20:77-82. CrossRef Medline

Lane MA, Fuller DD, White TE, Reier PJ (2008) Respiratory neuroplasticity and cervical spinal cord injury: translational perspectives. Trends Neurosci 31:538-547. CrossRef Medline

Lane MA, Lee KZ, Fuller DD, Reier PJ (2009) Spinal circuitry and respiratory recovery following spinal cord injury. Respir Physiol Neurobiol 169: 123-132. CrossRef Medline

Lepore AC, Tolmie C, O’Donnell J, Wright MC, Dejea C, Rauck B, Hoke A, Ignagni AR, Onders RP, Maragakis NJ (2010) Peripheral hyperstimulation alters site of disease onset and course in SOD1 rats. Neurobiol Dis 39:252-264. CrossRef Medline

Lepore AC, O’Donnell J, Kim AS, Williams T, Tuteja A, Rao MS, Kelley LL, Campanelli JT, Maragakis NJ (2011) Human glial-restricted progenitor transplantation into cervical spinal cord of the SOD1 mouse model of ALS. PLoS One 6:e25968. CrossRef Medline

Li K, Nicaise C, Sannie D, Hala TJ, Javed E, Parker JL, Putatunda R, Regan KA, Suain V, Brion JP, Rhoderick F, Wright MC, Poulsen DJ, Lepore AC (2014) Overexpression of the astrocyte glutamate transporter GLT1 exacerbates phrenic motor neuron degeneration, diaphragm compromise, and forelimb motor dysfunction following cervical contusion spinal cord injury. J Neurosci 34:7622-7638. CrossRef Medline

Li K, Javed E, Hala TJ, Sannie D, Regan KA, Maragakis NJ, Wright MC, Poulsen DJ, Lepore AC (2015) Transplantation of glial progenitors that overexpress glutamate transporter GLT1 preserves diaphragm function following cervical SCI. Mol Ther 23:533-548. CrossRef Medline 
Lu P, Jones LL, Tuszynski MH (2005) BDNF-expressing marrow stromal cells support extensive axonal growth at sites of spinal cord injury. Exp Neurol 191:344-360. CrossRef Medline

Lynskey JV, Sandhu FA, Dai HN, McAtee M, Slotkin JR, MacArthur L, Bregman BS (2006) Delayed intervention with transplants and neurotrophic factors supports recovery of forelimb function after cervical spinal cord injury in adult rats. J Neurotrauma 23:617-634. CrossRef Medline

Mantilla CB, Zhan WZ, Sieck GC (2004) Neurotrophins improve neuromuscular transmission in the adult rat diaphragm. Muscle Nerve 29:381386. CrossRef Medline

Mantilla CB, Gransee HM, Zhan WZ, Sieck GC (2013) Motoneuron BDNF/ TrkB signaling enhances functional recovery after cervical spinal cord injury. Exp Neurol 247:101-109. CrossRef Medline

Mantilla CB, Greising SM, Stowe JM, Zhan WZ, Sieck GC (2014a) TrkB kinase activity is critical for recovery of respiratory function after cervical spinal cord hemisection. Exp Neurol 261:190-195. CrossRef Medline

Mantilla CB, Stowe JM, Sieck DC, Ermilov LG, Greising SM, Zhang C, Shokat KM, Sieck GC (2014b) TrkB kinase activity maintains synaptic function and structural integrity at adult neuromuscular junctions. J Appl Physiol (1985) 117:910-920. CrossRef Medline

Martínez-Gálvez G, Zambrano JM, Diaz Soto JC, Zhan WZ, Gransee HM, Sieck GC, Mantilla CB (2016) TrkB gene therapy by adeno-associated virus enhances recovery after cervical spinal cord injury. Exp Neurol 276: 31-40. CrossRef Medline

Miller RG, Bryan WW, Dietz MA, Munsat TL, Petajan JH, Smith SA, Goodpasture JC (1996) Toxicity and tolerability of recombinant human ciliary neurotrophic factor in patients with amyotrophic lateral sclerosis. Neurology 47:1329-1331. CrossRef Medline

Neimert-Andersson T, Binnmyr J, Enoksson M, Langebäck J, Zettergren L, Hällgren AC, Franzén H, Lind Enoksson S, Lafolie P, Lindberg A, AlTawil N, Andersson M, Singer P, Grönlund H, Gafvelin G (2014) Evaluation of safety and efficacy as an adjuvant for the chitosan-based vaccine delivery vehicle ViscoGel in a single-blind randomised phase I/IIa clinical trial. Vaccine 32:5967-5974. CrossRef Medline

Nicaise C, Hala TJ, Frank DM, Parker JL, Authelet M, Leroy K, Brion JP, Wright MC, Lepore AC (2012) Phrenic motor neuron degeneration compromises phrenic axonal circuitry and diaphragm activity in a unilateral cervical contusion model of spinal cord injury. Exp Neurol 235:539552. CrossRef Medline

Nicaise C, Frank DM, Hala TJ, Authelet M, Pochet R, Adriaens D, Brion JP, Wright MC, Lepore AC (2013) Early phrenic motor neuron loss and transient respiratory abnormalities after unilateral cervical spinal cord contusion. J Neurotrauma 30:1092-1099. CrossRef Medline

Novikova LN, Novikov LN, Kellerth JO (2000) BDNF abolishes the survival effect of NT-3 in axotomized clarke neurons of adult rats. J Comp Neurol 428:671-680. CrossRef Medline

Novikova LN, Novikov LN, Kellerth JO (2002) Differential effects of neurotrophins on neuronal survival and axonal regeneration after spinal cord injury in adult rats. J Comp Neurol 452:255-263. CrossRef Medline

Perrier JF, Rasmussen HB, Christensen RK, Petersen AV (2013) Modula- tion of the intrinsic properties of motoneurons by serotonin. Curr Pharm Des 19:4371-4384. CrossRef Medline

Pitts EV, Potluri S, Hess DM, Balice-Gordon RJ (2006) Neurotrophin and trk-mediated signaling in the neuromuscular system. Int Anesthesiol Clin 44:21-76. Medline

Sasaki M, Radtke C, Tan AM, Zhao P, Hamada H, Houkin K, Honmou O, Kocsis JD (2009) BDNF-hypersecreting human mesenchymal stem cells promote functional recovery, axonal sprouting, and protection of corticospinal neurons after spinal cord injury. J Neurosci 29:14932-14941. CrossRef Medline

Scarano A, Carinci F, Piattelli A (2009) Lip augmentation with a new filler (agarose gel): a 3-year follow-up study. Oral Surg Oral Med Oral Pathol Oral Radiol Endod 108:e11-e15. CrossRef Medline

Shanmuganathan K, Gullapalli RP, Zhuo J, Mirvis SE (2008) Diffusion tensor MR imaging in cervical spine trauma. AJNR Am J Neuroradiol 29: 655-659. CrossRef Medline

Siegel CS, Fink KL, Strittmatter SM, Cafferty WB (2015) Plasticity of intact rubral projections mediates spontaneous recovery of function after corticospinal tract injury. J Neurosci 35:1443-1457. CrossRef Medline

Strakowski JA, Pease WS, Johnson EW (2007) Phrenic nerve stimulation in the evaluation of ventilator-dependent individuals with C4- and C5-level spinal cord injury. Am J Phys Med Rehabil 86:153-157. CrossRef Medline

Warren PM, Alilain WJ (2014) The challenges of respiratory motor system recovery following cervical spinal cord injury. Prog Brain Res 212:173220. CrossRef Medline

Warren PM, Awad BI, Alilain WJ (2014) Drawing breath without the command of effectors: the control of respiration following spinal cord injury. Respir Physiol Neurobiol 203:98-108. CrossRef Medline

Weishaupt N, Blesch A, Fouad K (2012) BDNF: the career of a multifaceted neurotrophin in spinal cord injury. Exp Neurol 238:254-264. CrossRef Medline

Wright MC, Son YJ (2007) Ciliary neurotrophic factor is not required for terminal sprouting and compensatory reinnervation of neuromuscular synapses: re-evaluation of CNTF null mice. Exp Neurol 205:437-448. CrossRef Medline

Wright MC, Cho WJ, Son YJ (2007) Distinct patterns of motor nerve terminal sprouting induced by ciliary neurotrophic factor vs. botulinum toxin. J Comp Neurol 504:1-16. CrossRef Medline

Wright MC, Potluri S, Wang X, Dentcheva E, Gautam D, Tessler A, Wess J, Rich MM, Son YJ (2009) Distinct muscarinic acetylcholine receptor subtypes contribute to stability and growth, but not compensatory plasticity, of neuromuscular synapses. J Neurosci 29:14942-14955. CrossRef Medline

Ye JH, Houle JD (1997) Treatment of the chronically injured spinal cord with neurotrophic factors can promote axonal regeneration from supraspinal neurons. Exp Neurol 143:70-81. CrossRef Medline

Zimmer MB, Nantwi K, Goshgarian HG (2007) Effect of spinal cord injury on the respiratory system: basic research and current clinical treatment options. J Spinal Cord Med 30:319-330. CrossRef Medline 